Accepted for publication in the Astrophysical Journal on 13th of April 2012

\title{
An upscattering spectral formation model for the prompt emission of Gamma-Ray Bursts
}

\author{
Lev Titarchuk ${ }^{1,2,4}$, Ruben Farinelli ${ }^{1}$, Filippo Frontera ${ }^{1}$ and Lorenzo Amati ${ }^{3}$,
}

\begin{abstract}
We propose a model for the spectral formation of Gamma Ray Burst (GRB) prompt emission, where the phenomenological Band's function is usually applied to describe the GRB prompt emission. We suggest that the GRB prompt emission is mainly a result of two upscattering processes. The first process is the Comptonization of relatively cold soft photons of the star off electrons of a hot shell of plasma of temperature $T_{e}$ of the order of $10^{9} \mathrm{~K}$ ( or $k T_{\mathrm{e}} \sim 100 \mathrm{keV}$ ) that moves sub-relativistically with the bulk velocity $V_{\mathrm{b}}$ substantially less than the speed of light $c$. In this phase, the Comptonization parameter $Y$ is high and the interaction between a blackbody-like soft seed photon population and hot electrons leads to formation of a saturated Comptonization spectrum modified by the sub-relativistic bulk outflow. The second process is an upscattering of the previously Comptonized spectrum by the plasma outflow once it becomes relativistic. This process gives rise to the high-energy power-law component above the peak in the $E F(E)$-diagram where $F(E)$ is the energy flux. The latter process can be described by a convolution of the Comptonized spectrum with a broken-power-law Green function. Possible physical scenarios for this second upscattering process are discussed. In the framework of our model, we give an interpretation of the Amati relation between the intrinsic spectral peak photon energy and radiated energy or luminosity, and we propose a possible explanation of the GRB temporal variability.
\end{abstract}

Subject headings: radiation mechanisms: general — radiative transfer - gammaray burst: general — stars: massive

\footnotetext{
${ }^{1}$ Dipartimento di Fisica, Università di Ferrara, via Saragat 1, 44122 Ferrara, Italy, email:titarchuk@fe.infn.it

${ }^{2}$ George Mason University, Fairfax, VA 22030, USA, email: ltitarch@gmu.edu

${ }^{3}$ INAF-IASF, Sezione di Bologna, via Gobetti 101, 40129 Bologna, Italy

${ }^{4}$ NASA/Goddard Space Center, Greenbelt, 20770, USA, email: lev@milkyway.gsfc.nasa.gov
} 


\section{Introduction}

Understanding the physical processes which give rise to the observed spectra of the prompt emission of Gamma Ray Bursts (GRB) is presently one of the most exciting issues studied by both the theoretical and observational community. The Band function (Band et al. 1993) up to now widely used to describe their prompt emission is a pure phenomenological model. It consists of two low-energy and high-energy powerlaws with photon index $\Gamma_{1}$ and $\Gamma_{2}$, respectively, smoothly joined at some energy $E_{\mathrm{b}}$. Among the physical models proposed for a possible origin of the Band function it is worth mentioning the optically-thin synchrotron model (e.g. Tavani 1996), in which the electron population of the relativistically expanding shell is accelerated by internal shock collisions, eventually producing a supra-thermal powerlaw-like distribution in the tail of the Maxwellian. This mixed electron population interacts with possible turbulent magnetic fields frozen in the plasma emitting synchrotron photons and forming synchrotron spectra (Rees \& Mészáros 2005). The observed break in the energy distribution is thus naturally explained by the transition from the optically thick to optically thin emission regime. However the current spectral analysis of both time-integrated (e.g., Crider et al. 1997 ) and time-resolved (e.g., Crider et al. 1998 ; Frontera et al. 2000; Ghirlanda et al. 2003) prompt GRB spectra has revealed some problems related to the synchrotron/SSC emission models.

Many theoretical efforts have also been performed in the thermal (photospheric) interpretation (e.g., Thompson 1994; Mészáros \& Rees 2000; Beloborodov 2010; Toma et al. 2011). From the observational point of view, Ryde \& Pe'er (2009, hereafer RP09), analyzed a sample of GRBs using archival BATSE data and found that the time-resolved spectra can be fit by a high temperature $\left(10^{9} \mathrm{~K}\right)$ blackbody $(\mathrm{BB})$ spectrum plus a powerlaw $(\mathrm{PL})$. Their model, albeit phenomenological, actually strongly points in favor of the presence of a photospheric emission process at the origin of almost $50 \%$ of the total emitted energy. In fact, the low-energy threshold of BATSE prevented RP09 to use more detailed thermal models, but the good fits provided by application of the BB+PL model could point in favor of the presence of a photospheric (Compton-saturated) emission plus a second process giving rise to the PL-like hard X-ray emission. As also argued by RP09, one of the advantages in considering thermal processes is to reduce the kinetic-to-radiation conversion efficiency, which is difficult to account by other theoretical models. However recently Zhang \& Yan (2011) demonstrate that the observed high efficiency is a drawback of the internal shock model, but is not an issue for the models that invoke dissipation of a Poynting flux.

Another issue related to the GRB origin is the nature of the progenitor. Long GRBs

are preferably observed at high redshift, with an average $z>2$ (Jakobsson et al. 2006; Fiore et al. 2007). They are concentrated in small, irregular galaxies and show strong 
evidence of association with Type Ic supernovae (Kelly et al. 2008). Both theoretical and observational works point in favor of a Wolf-Rayet star with mass higher than $\sim 40 M_{\odot}$ (Raskin et al. 2008) and stripped H-envelope as the most likely progenitor of long GRBs (the collapsar model, Woosley 2011). These progenitor masses are expected to leave, in their explosion, a central remnant and it is generally supposed that the GRB engine is powered by gravitational energy release of a torus of matter debris accreting onto a black hole. In some sense, a temporary microquasar forms in the star after core-collapse, and consequently torus accretion may power an expanding (relativistic) jet. However, unlike the case of AGN or microquasar jets, the environment surrounding the outflow is not the interstellar medium but the star photosphere which may play a significant role in dragging the jet kinetic energy. The confinement level of the expanding jet depends on the external environment; if the condition $\theta_{\mathrm{J}} M_{\mathrm{J}} \lesssim 2$ (where $\theta_{\mathrm{J}}$ and $M_{\mathrm{J}}$ are the jet opening angle and Mach number, respectively) is satisfied, the pressure equilibrium with the surroundings and jetstructure is almost maintained, while in the opposite case strong shocks inside the jet may form and the overpressure allows a free expansion (Gehrels et al. 2012). Baryon-loading may also be a key ingredient in the determination of the jet bulk velocity.

In this context, it is worth mentioning the theoretical work by Lazzati et al. (2009), which used a numerically relativistic code (Fryxell et al. 2000) to evaluate the evolution of a jet as it leaves a massive progenitor star after core-collapse and propagates to the photospheric radius, where radiation is eventually released. In their simulations, Lazzati et al. (2009) considered a $16 M_{\odot}$ Wolf-Rayet progenitor star evolved to pre-explosion and a jet with initial opening angle $\theta_{0}=10^{\circ}$ and Lorentz factor $\gamma_{0}=5$ at a distance $R=10^{9} \mathrm{~cm}$ from the center of the star. Although limited to this particular configuration, the authors showed that the theoretical light curves are in good agreement with the observed ones. In addition, the photospheric temperatures are in the range 100-300 keV, in turn leading to values of the rest-frame peak energy $E_{\mathrm{p}}$ consistent with observations. However, also progenitors associated with much more massive stars (Population III stars) cannot be excluded, at least for farthest GRBs. Indeed, supernovae associated with these stars with Helium core $M_{\mathrm{c}}>100 M_{\odot}$, have been observed also in the closer Universe (Gal-Yam et al. 2009). Such progenitors, with initial mass in the range 130-260 $M_{\odot}$, have been shown to explode due to pair-instability (PI) (e.g., Langer et al. 2007; Woosley et al. 2007; Woosley 2011) with no compact remnant. Thus GRBs could be the result of helium burning in degenerated conditions, in which the burning occurs in deflagration regime [see details in Chardonnet et al. (2010)]. Also in this case, (e.g., Imshennik et al. 1999; Bychkov et al. 2006) have shown that a shock runs away from the burning (reaction) zone which leads to the development of an outflow (jet-like) structure. In terms of this model, the GRB spectrum would be originated from Compton upscattering of soft photons (probably $\sim 1 \mathrm{keV}$ ) in the hot sub-relativistic bulk 
outflow region. The hydrodynamical simulations of Chardonnet et al. (2010) show that the electron temperature of the hot corona region can be as high as $T_{\mathrm{e}} \sim 10^{9} \mathrm{~K}$.

The rapid temporal variability on time scale of order $\Delta T \lesssim 1 \mathrm{~s}$ and shorter, observed during the GRB prompt phase, implies that the sources are compact with size $R<c \Delta T \sim$ $10^{11} \mathrm{~cm}$. To avoid the problem of high compactness, that would imply high optical depth for pair production $\tau_{\gamma \gamma} \gg 1$, a high Lorentz factor of the relativistic motion of the emitting plasma is the standard scenario (Piran et al. 1999). Alternatively, to decrease $\tau_{\gamma \gamma}$, it is needed to consider a much more extended volume over which the photon field is distributed.

In this Paper we offer a model to explain the GRB prompt spectral formation in the context of a photospheric scenario in which the main process is the Comptonization of the relatively soft photons of the star by a hot subrelativistic outflow within an area close to the photospheric radius (optical depth of 3-5) likely symmetric with respect to the rotational axis of the star.

We start from the theoretical and numerical results of the Comptonization problem reported in Titarchuk et al. (1997, hereafter TMK97) and Farinelli et al. (2008, hereafter F08), but for the case of an early subrelativistic bulk outflow phase produced during the supernova explosion. This physical scenario is natural in the case of PI-SNe and it is foreseen in the case of an electromagnetic outflow (Lyutikov et al. 2003), while in the collapsar model, the jet formed inside the star is assumed to be initially relativistic. Whether this condition can hold, may depend on several parameters, such as the initial jet energy, the chemical composition of the star envelope (in particular the presence or not of a H-envelope), the core angular momentum and the influence of magnetic torques (Gehrels et al. 2012; Woosley 2011).

In $\$ 2$ we present the radiative transfer mathematical formalism, necessary to investigate both the spectral formation in the subrelativistic phase, and the formation of the highenergy PL component. In $\S 3$ we demonstrate that our upscattering model of GRB radiation reproduces the Amati relation between the $E F(E)$ peak energy $E_{p}$ and the GRB radiated energy $E_{i s o}$ or luminosity $L_{g r b}$. In $\$ 4$ we put arguments for the observed high and slow temporal variabilities of GRB emission. In $\$ 5$ we estimate the resulting luminosity of GRB, discuss a formation of the high-energy PL component and our scenario in the light of the Fermi results. In $₫ 6$ we draw our conclusions. 


\section{Upscattering of soft photons of the star in the hot plasma (Compton) cloud}

\subsection{Spectral formation in sub-relativistic outflow}

The emergent spectrum produced by Comptonization of soft photons off a thermal electron population in the presence of bulk motion has been first formulated by Blandford \& Pavne (1981, hereafter BP81) and later investigated by several authors (e.g., Cowsik \& Lee 1982; Colpi 1988). Depending on the sign of the divergence of the velocity field, the resulting spectrum can be harder $(\nabla \cdot \mathbf{V}<0)$ or softer $(\nabla \cdot \mathbf{V}>0)$ with respect to the case of

static medium (Sunyaev \& Titarchuk 1980, hereafter ST80). Examples of the first case (converging flow) are reported in TMK97 and F08, while the second case (diverging flow) was investigated by Laurent \& Titarchuk (1999, 2007, hereafter LT07).

The basic idea of the photon scattering effect in the case of bulk inflow and outflow is presented in Fig. 1 of LT07: a photon emitted outwards near inner boundary and then scattered at a certain point by an electron moving with velocity $\mathbf{V}_{1}$, is received by an electron having velocity $\mathbf{V}_{2}$ with frequency $\nu_{2}=\nu_{1}\left[1+\left(\mathbf{V}_{1}-\mathbf{V}_{2}\right) \cdot \mathbf{n} / c\right]$ where $\mathbf{n}$ is a unit vector along the path of the photon at the scattering point. In a diverging flow $\left(\mathbf{V}_{1}-\mathbf{V}_{2}\right) \cdot \mathbf{n} / c<0$ and photons are successively redshifted, until they are scattered to an observer at infinity. On the other hand, in a converging flow $\left(\mathbf{V}_{1}-\mathbf{V}_{2}\right) \cdot \mathbf{n} / c>0$ and photons are blueshifted. This pure geometrical (Doppler) effect can be analytically described when the electron temperature is very low, as the contribution of the thermal velocity vector $\mathbf{V}_{\text {th }}$ is negligible compared with the dynamical (bulk) velocity vector $\mathbf{V}_{\mathrm{b}}$. If, on the other hand, the plasma temperature is high enough so that $\mathbf{V}_{\mathrm{b}}$ and $\mathbf{V}_{\mathrm{th}}$ become comparable, then the emergent Comptonization spectrum is marginally modified by the presence of the bulk motion. A detailed analytical treatment of photon diffusion (Fokker-Planck approximation) in a converging flow is reported in TMK97 and F08. Below we will start from the same equations treated by these authors, but we will consider the case of a fluid which is subrelativistically expanding (diverging flow), namely we consider a velocity field with $\nabla \cdot \mathbf{V}_{\mathrm{b}}>0$.

\subsection{Formulation of the bulk motion problem. The main equation and its solution}

In this section, we provide the mathematical formulation of the problem required to compute the emerging spectrum due to Comptonization of soft photons by electrons in a hot corona bounced to the top of the star and moving outwardly with subrelativistic velocity (see Fig. 1). The spectrum can be derived using the Fokker-Planck expansion of the radiative transfer equation in the presence of bulk motion (BP81, see also Eq. 13 in TMK97): 


$$
\begin{gathered}
\frac{\partial n}{\partial t}+\mathbf{V}_{\mathrm{b}} \cdot \nabla n=\nabla \cdot\left[\frac{c}{3 k} \nabla n\right]+\frac{1}{3} \nu \frac{\partial n}{\partial \nu} \nabla \cdot \mathbf{V}_{\mathrm{b}}+ \\
\frac{1}{\nu^{2}} \frac{\partial}{\partial \nu}\left[\frac{k h}{m_{\mathrm{e}} c} \nu^{4}\left(n+\frac{k T_{\mathrm{e}}+m_{\mathrm{e}} V_{\mathrm{b}}^{2} / 3}{h} \frac{\partial n}{\partial \nu}\right)\right]+j(r, \nu),
\end{gathered}
$$

where $\mathbf{V}_{\mathrm{b}}$ is the bulk velocity field of the outflow, $k T_{\mathrm{e}}$ its electron temperature, $k(r)=$ $N_{\mathrm{e}}(r) \sigma_{T}$ is the inverse of the scattering mean free path, $n \equiv n(r, \nu)$ is the zero-moment occupation number of the specific intensity and $j(r, \nu)$ is the source term.

Let $N(r)=N_{0}\left(r_{0} / r\right)^{\mu}$ be the radial number density profile of the bulk outflow, with radial outward velocity given by

$$
\beta(r)=\beta_{0}\left(\frac{r_{0}}{r}\right)^{2-\mu},
$$

which is easily derived from the continuity equation in spherical geometry. Here $\beta_{0}=V_{0} / c$ is the dimensionless outflow velocity with respect to speed of light at the bottom of the shell. Thomson optical depth of the flow from some radius $r$ to infinity is given by

$$
\tau=\int_{r}^{\infty} N_{\mathrm{e}} \sigma_{T} d r^{\prime}=\tau_{0}\left(r_{0} / r\right)^{\mu-1}
$$

where $\tau_{0}$ is the optical depth at the bottom of the shell. Using the given radial density profile together with definitions (2) and (3), the steady-state Fokker-Plank (diffusion) equation (1) becomes

$$
\frac{1}{\Theta} L_{\tau} n+\delta \cdot x \frac{\partial n}{\partial x}+\frac{1}{x^{2}} \frac{\partial}{\partial x}\left[x^{4}\left(f_{\mathrm{b}} \frac{\partial n}{\partial x}+n\right)\right]=-\frac{j(\tau, x)}{\Theta k}
$$

where

$$
\delta=\frac{\mu}{3 \Theta(\mu-1)} \frac{\beta_{0}}{\tau_{0}}\left(\frac{\tau}{\tau_{0}}\right)^{(3-2 \mu) /(\mu-1)} .
$$

In equation (44), $L_{\tau}$ is the space operator (see Titarchuk et al. 2003), $x \equiv h \nu /\left(m_{\mathrm{e}} c^{2}\right)$ and $\Theta \equiv k T_{\mathrm{e}} /\left(m_{\mathrm{e}} c^{2}\right)$ are dimensionless photon energy and electron temperature, respectively, while $f_{\mathrm{b}}=1+\left(V_{\mathrm{b}} / c\right)^{2} /(3 \Theta)$.

We note that $\delta$ in equation (5) is constant only for $\mu=3 / 2$, which corresponds to the free-fall velocity profile case (BP81), while for $\mu \neq 3 / 2$ it is a function of the optical depth $\tau$. For the particular case of constant outflow velocity $(\mu=2)$, equation (4) becomes

$$
\frac{1}{\Theta}\left[\frac{1}{3} \frac{\partial^{2} n}{\partial \tau^{2}}-\left(\frac{4}{3 \tau}-\beta_{0}\right) \frac{\partial n}{\partial \tau}\right]+\frac{2 \beta_{0}}{3 \tau} x \frac{\partial n}{\partial x}+\frac{\partial}{\partial x}\left[x^{4}\left(f_{\mathrm{b}} \frac{\partial n}{\partial x}+n\right)\right]=-\frac{j(\tau, x)}{\Theta k}
$$


The dependence of $\delta$ on $\tau$ does not allow to find a solution of the equation (6) with the method of variable separation (space and energy). However, LT07 demonstrate that for a constant velocity profile, $\delta$ can be replaced by its value at some effective optical depth $\tau_{\text {eff }}$, which is a fraction of total optical depth $\tau_{0}$ of the expanding shell. Thus, replacing $\tau$ with $\tau_{\text {eff }}$ in equation (5) we can now rewrite equation (4) in the form

$$
\left(\mathcal{L}_{\tau}+L_{x}\right) n(x, \tau)=-\frac{\varphi(x)}{x^{3}} s(\tau)
$$

where $L_{x}$ and $\mathcal{L}_{\tau}=\Theta^{-1} L_{\tau}$ are the energy and space differential operators (see these operators in Eq. 4 for $\delta=$ const and Eq. [6 respectively). $\varphi(x)$ is the occupation number of the seed photons, and $s(\tau)$ their spatial distribution in the bounded medium. The solution of equation (7) can be conveniently expressed as a series $n(x, \tau)=\sum c_{k} N_{k}(x) R_{k}(\tau)$, where $R_{k}(\tau)$ are the eigenfunctions of the space operator $L_{\tau}$ and $c_{k}$ are the expansion coefficients over the seed photon spatial distribution $s(\tau)$. We calculate the Comptonization spectrum using only the first term $k=1$ of the series (TMK97), which is obtained solving the equation

$$
\begin{array}{r}
f_{\mathrm{b}} x^{2} \frac{d^{2} N(x)}{d^{2} x}+\left(x^{2}+4 f_{\mathrm{b}} x+\delta x\right) \frac{d N(x)}{d x} \\
+(4 x-\gamma) N(x)=-\gamma \frac{\varphi(x)}{x^{3}}
\end{array}
$$

where

$$
\delta=\frac{2 \beta_{0}}{3 \tau_{\mathrm{eff}} \Theta}
$$

is given for $\mu=2, \tau_{\text {eff }}=<\tau>$ (see Eq. 5) and $\gamma=\lambda_{1}^{2} / \Theta$. Here $\lambda_{1}^{2}$ is the first eigenvalue of the space operator, $L_{\tau}$ specifically

$$
L_{\tau} R(\tau)+\lambda_{1}^{2} R(\tau)=0
$$

For the particular case $\left(V_{\mathrm{b}} / c\right)^{2} \ll 3 \Theta, f_{\mathrm{b}} \sim 1$ and it is possible to express the analytical solution of equation (8) in terms of the convolution of the seed photon spectrum $\varphi(x)$ with the Green function (GF) of the energy operator $L_{x}$ according to

$$
F_{\mathrm{tb}}(x)=\int_{0}^{\infty} G\left(x, x_{0}\right) \varphi\left(x_{0}\right) d x_{0}
$$

where the GF is defined as

$$
G\left(x, x_{0}\right)=\frac{C_{\mathrm{N}} e^{-x}}{x_{0} \Gamma(q)}\left\{\begin{array}{l}
\left(\frac{x}{x_{0}}\right)^{\alpha+3-\delta}{ }_{1} F_{1}(\alpha, q, x) I\left(x_{0}, \alpha, \delta\right), \text { for } x \leq x_{0} \\
\left(\frac{x}{x_{0}}\right)^{-\alpha}{ }_{1} F_{1}\left(\alpha, q, x_{0}\right) I(x, \alpha, \delta), \text { for } x \geq x_{0},
\end{array}\right.
$$


where

$$
I(x, \alpha, \delta)=\int_{0}^{\infty} e^{-t}(x+t)^{\alpha+3-\delta} t^{\alpha-1} d t
$$

and

$$
q=2 \alpha+4-\delta,
$$

while ${ }_{1} F_{1}(a, b, z)$ is the Kummer confluent hypergeometric function. Note the change of sign of $\delta$ with respect to the same GF reported in F08 because here we are considering a diverging flow. The energy spectral index $\alpha$ of the GF is given by

$$
\alpha=-\frac{3-\delta}{2}+\left[\left(\frac{\delta+3}{2}\right)^{2}+\gamma\right]^{1 / 2},
$$

and the normalization $C_{\mathrm{N}}=\alpha(\alpha-\delta+3)$ is chosen in order to conserve the total photon number, namely

$$
\int_{0}^{\infty} G\left(x, x_{0}\right) \frac{d x}{x}=\frac{1}{x_{0}} .
$$

It is worth pointing out that $\lambda_{1}^{2} \propto 1 / \tau_{0}^{2}$, where $\tau_{0}$ is the total Thomson optical depth of the shell, so that $\gamma \ll 1$ corresponds to $\Theta \tau_{0}^{2} \gg 1$, namely for the case of saturated Comptonization. In the case of a pure thermal motion $(\delta=0)$ this condition implies $\alpha \sim 0$ (see Eq. [15), and the emergent spectrum obtained from equation (11) has a Wien-like shape, no matter which is the spectral distribution $\varphi(x)$ of the seed photons. On the other hand, if bulk motion is present, then $\alpha \approx \delta$.

In Figure 2 we present a typical example of the shape of the GF for different values of the bulk parameter $\delta$ defined in equation (9), for the case of soft photon monochromatic injection $\left(x_{0}=0.1\right)$ and $\gamma \ll 1$. While in Figure 3 we show a convolution of the Green function with a BB seed spectrum (see Eq. 11). It is evident that the important role is played by bulk Comptonization in diverging flow in determining the slope of the high-energy wing and the position of the high-energy cut-off, both quantities modify the position of the peak in the $\mathrm{EF}(\mathrm{E})$ spectrum (see Fig. 1 in $\mathrm{F} 08$ for comparison with the case of converging flow).

As already outlined above, these analytical results can be obtained for the case $f_{\mathrm{b}}=1$ (see Eq. 8). On the other hand, if $f_{\mathrm{b}}>1$, the equation has to be solved numerically, and we report the details of the numerical solution in Appendix $\mathrm{A}$. 


\subsection{High energy power-law component in the GRB prompt emission}

The solution of equation (8) clearly shows that above the energy peak $E_{\mathrm{p}}$ in the $\mathrm{EF}(\mathrm{E})$ diagram, the spectrum has an exponential rollover (see Fig. 3). This spectral feature is characteristic of Comptonization both in a pure thermal or subrelativistic moving cases. For a thermal Comptonization, the cut-off energy $E_{\mathrm{c}}$ is dictated only by the plasma electron temperature, with $E_{\mathrm{c}}>2 k T_{\mathrm{e}}$, while when bulk motion is present (inflow or outflow), the bulkparameter $\delta$ also concurs in dictating the $E_{\mathrm{c}}$-value (see Figs. 2 and 3). In both cases, the high-energy exponential rollover arises from the presence of the thermal recoil-effect $\propto N(x)$ in the radiative transfer equation modified by the term $\propto \delta$ (see Eq. 4).

However, it is a well established observational result that most (albeit not all) of the observed prompt GRB spectra show an extended PL above the peak energy in the $E F(E)$-diagram [where $F(E)$ is the energy spectrum], which is phenomenologically described by the second index $\Gamma_{2}$ of the Band function. This high-energy feature cannot be reproduced by the solution of Equation (8), and a second component to describe the broadband spectrum is required. One possibility is to simply add a PL to the thermal spectral component, as was done, e.g., by RP09, who fitted the BATSE spectra with a BB+PL model. From a physical point of view, this model can imply a different origin for the apparently thermal and non-thermal part of the GRB spectrum. It is also well known that the inclusion of a simple PL in broad-band spectral model can introduce some biases, because this component, intended to be used for describing only part of the spectrum (usually at high energies), actually covers the whole spectral range, producing undesired effects such as overestimation of the flux at low energies (the effect getting stronger for steeper photon index Г) (Frontera et al. 2012, in preparation; Ghirlanda et al. 2007).

Here, we investigate a different possibility; there is a general theorem that the solution of an equation with linear differential operator and a source term (such as Eq. 8) is obtained by the convolution of the GF of the given operator with the source function itself, according to Eq. (11). Examples for this convolution, in addition to the results of previous section, are reported for the case of pure thermal Comptonization (ST80), thermal plus bulk Comptonization (TMK97, F08), and Comptonization in strong-magnetic field (Becker et al. 2007). The resulting energy spectrum can be written in the general form as

$$
F(E)=\frac{1}{A+1}\left[\varphi(E)+A \times \varphi\left(E_{0}\right) * G\left(E, E_{0}\right)\right]
$$

where the two terms on the right-hand side represent the direct and Comptonized (convolved) part of the seed spectrum, respectively. The weighting factor $A$ is related to the physical and geometrical configuration of the system. The high-energy behavior of the spectrum depends on the kernel $G\left(E, E_{0}\right)$; for example, if it is a broken PL with no cut-off, then the convolution 
with $\varphi(E)$ (see Eq. 11) produces a PL (BMC model in the XSPEC package).

Thus the observed GRB prompt spectra, phenomenologically described by the Band function, can be obtained by an equation of the form (17) with $A \gg 1$, the source term $\varphi(E)$ obtained by the solution of equation (8) and using a broken-PL GF with no recoil-term. The latter can be conveniently described by the following form:

$$
G_{b p l}\left(x, x_{0}\right)=\frac{\alpha_{\mathrm{b}}\left(\alpha_{\mathrm{b}}+3\right)}{x_{0}\left(2 \alpha_{\mathrm{b}}+3\right)} \begin{cases}\left(\frac{x}{x_{0}}\right)^{\alpha_{\mathrm{b}}+3} & \text { for } x \leq x_{0} \\ \left(\frac{x}{x_{0}}\right)^{-\alpha_{\mathrm{b}}} & \text { for } x \geq x_{0}\end{cases}
$$

where the subscript $b$ has been used to avoid confusion with the index $\alpha$ of the GF related to the thermal plus bulk energy operator (Eq. 12). Note that this is the GF of the energy operator in equation (4) with $f_{\mathrm{b}}=1, \delta=0$ and the recoil term $\propto x^{4} N$ in square brackets dropped (Sunyaev \& Titarchuk 1980), which leads to the equation

$$
x^{2} \frac{d^{2} W}{d x^{2}}+4 x \frac{d W}{d x}-\alpha_{\mathrm{b}}\left(\alpha_{\mathrm{b}}+3\right) W(x)=-\alpha_{\mathrm{b}}\left(\alpha_{\mathrm{b}}+3\right) \varphi(x),
$$

where $\varphi(x)=N(x)$ is obtained by solving equation (8). However, the spectrum obtained as a solution of equation (8) has no analytical representation, so performing a numerical convolution according to equation (11) where $G_{b p l}\left(x, x_{0}\right)$ is given by equation (18) and $\varphi(x)$ by $N(x)$ in equation (8) can be time-consuming in terms of CPU computation. We thus again numerically solved equation (19) using a finite-difference method. From the mathematical point of view, there is perfect equivalence between the analytical solutions given by the convolution above described and direct numerical solution of the equation. In our model $\alpha_{b}$ is a free parameter, related to the high-energy PL photon index $\Gamma_{2}$ of the Band function, by $\alpha_{b}=-\Gamma_{2}-1$.

To better explain this behavior, in Fig. 4 we show two spectra obtained from the BMC model of the XSPEC package (TMK97), where the source function $\varphi(E)$ is assumed to be a $\mathrm{BB}$ occupation number. Note that when $A \gg 1$, only the second (convolution) term contributes to the spectral formation, and the low-energy and high-energy parts of the spectrum smoothly join at the peak energy, in a qualitative way which is very similar to the Band function.

This is actually the approach we adopted to develop our model: we first solved the Comptonization equation in the presence of subrelativistic bulk motion (see Eq. 8) and then we convolved it with the broken-PL GF of equation (18), setting $A \gg 1$ in equation (17). In Appendix $\mathrm{B}$ we describe the numerical methods used to address these issues. 


\section{The Amati's relation and its interpretation}

In spite of the still open discussion about the impact of possible selection effects on the correlation between the peak energy of $E F(E)$ diagram $E_{p}$ and the GRB radiated energy or luminosity (Amati relation, AR, Amati et al. 2002), it is a matter of fact that all GRBs with known redshifts, but one (GRB 980425), follow this relation [see, e.g., Amati et al. (2009) and references therein]. In addition, it is found that also within single GRBs, this relation holds (Frontera et al. submitted; Ghirlanda et al. 2010).

Thus it is crucial to understand the origin of this relation, namely which is the mechanism (at first glance universal) which gives rise to the relation $E_{\mathrm{p}} \propto E_{\text {iso }}^{1 / 2}$ where $E_{\mathrm{p}}$ and $E_{\text {iso }}$ are the peak energy of $E F(E)$ diagram and isotropic radiated energy (fluence) of GRB respectively during the prompt phase.

The main issue to be investigated is to check how the parameters of our model concur in determining the energy peak value $E_{\mathrm{p}}$ in the $E F(E)$ diagram and the total luminosity $L_{\text {grb }}$ which integral over prompt emission time is $E_{\text {iso }}$.

In Fig. 5 we show the resulting spectra obtained by fixing the parameters of the early sub-relativistic phase and changing the spectral index $\alpha_{\mathrm{b}}$ of the GF defined by equation (18), which is responsible for the high-energy PL component. Given that $E_{\mathrm{p}}$ very slightly depends (within a factor less than two) on different $\alpha_{\mathrm{b}}$-values, we fix $\alpha_{\mathrm{b}}$ about 1.5. In Fig. 6] we show the theoretical $\mathrm{EF}(\mathrm{E})$ spectra obtained using our model alternatively changing $\beta_{0}, k T_{\mathrm{e}}, \delta$ and $k T_{\mathrm{bb}}$, namely the parameters which characterize the subrelativistic phase. In addition we also show an example of the spectrum obtained using the solution of Eqs. (8) and (19) compared them with the Band function in Fig. 7. The result is that the leading quantity in influence of $E_{\mathrm{p}}$ is the electron temperature $k T_{\mathrm{e}}$. This result is not surprising because it is evident that in any photospheric model with spectral emission dominated by Comptonization, the electron temperature represents a key ingredient, both in a pure thermal and dynamical cases (for $\beta \ll 1)$. On the other hand, it is worth pointing out that $E_{\mathrm{p}}$ is independent of values of the BB-like seed photon temperature $k T_{\mathrm{bb}}$. Thus different values of $k T_{\mathrm{bb}}$ do not change $E_{\mathrm{p}}$, but they determine the total (resulting) luminosity, as we discuss below.

The question that naturally arises is whether the observed dependence of $E_{\mathrm{p}}$ on $E_{\text {iso }}$

(or on luminosity $L_{\mathrm{grb}}$ ) and its intrinsic dispersion (Amati et al. 2008) are the result of a multi-parametric combination of the subrelativistic phase plus the effect of the non-thermal process or is the fundamental effect of $\gamma$ - ray emission.

As an example, in Figure 8 we report the results of the fit using our model (see Appendix B) of the time averaged prompt emission spectrum of GRB 990705 obtained with BeppoSAX and, for comparison, with the Band function. With our model, the best-fit parameters 
are $k T_{\mathrm{bb}}=1.8_{-1.4}^{+1.3} \mathrm{keV}, k T_{\mathrm{e}}=94_{-10}^{+30} \mathrm{keV}, \delta=0.32_{-0.03}^{+0.09}, \alpha_{b}=1.50_{-0.17}^{+0.40}$ and photospheric radius $R_{\mathrm{ph}}=1.5 \times 10^{13} \mathrm{~cm}$, with $\chi^{2} / \mathrm{dof}=168 / 182$. We fixed $\gamma=5 \times 10^{-3}$ (as a $\gamma$-value strictly equals to zero produces computer underflow) and outflow velocity $\beta_{0}=0.2$. The Band function on the other hand provides a low energy photon index $\Gamma_{1}=-0.86_{-1.24}^{+3.91}$ and $E_{0}=292_{-22}^{+18} \mathrm{keV}$ with $\chi^{2} / \mathrm{dof}=167 / 182$. In this case however the high-energy index is not constrained and we fixed $\Gamma_{2}=-2.5$ (note that because of sign convention $\alpha_{\mathrm{b}}=-\Gamma_{2}-1$ ).

The small values of the best fit parameters of our model $\gamma$ and $\delta$ correspond to the case of the saturated Comptonization for which the resulting index $\alpha \ll 1$ (see Eq. 15). Sunvaev \& Titarchuk (1980), Sunvaev \& Titarchuk (1985) [and see also Chakrabarti \& Titarchuk (1995) ] derive the formula for the Comptonization enhancement factor $\eta_{\text {comp }}$ which is a ratio of the resulting luminosity, that is in our case, the GRB luminosity $L_{\text {grb }}$ to the injected luminosity of soft photons $L_{\text {soft }}$. Namely

$$
\eta_{\mathrm{comp}}=\frac{L_{\mathrm{grb}}}{L_{\mathrm{soft}}}=q_{x_{0}}(\alpha) x_{0}^{\alpha-1},
$$

where

$$
q_{x_{0}}(\alpha)=\frac{\alpha(\alpha+3) \Gamma(\alpha+4) \Gamma(\alpha) \Gamma(1-\alpha)}{\Gamma(2 \alpha+4)}\left(1-x_{0}^{1-\alpha}\right),
$$

and $x_{0}=2.7 k T_{\mathrm{bb}} / k T_{\mathrm{e}}$. Thus when $\alpha \ll 1$

$$
\eta_{\text {comp }} \propto k T_{\mathrm{e}}
$$

But the flux of soft photons illuminating the hot spot (Compton cloud) $L_{\text {soft }}$ is

$$
L_{\mathrm{soft}}=\pi B_{\mathrm{soft}} S_{\mathrm{hs}}
$$

where $B_{\text {soft }}$ is the intensity of the blackbody radiation of the star and $S_{\mathrm{hs}}$ is the surface area of Compton hot spot.

The thermal wave propagates in the hot spot with plasma velocity $V_{p}$ whose value can change from one GRB to another.

As a consequence for each GRB $S_{\mathrm{hs}} \propto\left(V_{p} t\right)^{2}$ and then

$$
S_{\mathrm{cc}} \propto V_{p}^{2} \propto k T_{\mathrm{p}}=k T_{\mathrm{e}}
$$

Thus the luminosity of the GRB hot spot $L_{\text {grb }}$ should be

$$
L_{\mathrm{grb}}=\eta_{\mathrm{comp}} L_{\mathrm{soft}} \propto\left(k T_{\mathrm{e}}\right)^{2} .
$$


In order to calculate the GRB fluence $E_{\text {iso,grb }}$ one should integrate $L_{\text {grb }}$ over the GRB prompt emission time $T_{p r}$, namely

$$
E_{\text {iso }}=\int_{0}^{T_{p r}} L_{\mathrm{grb}}(t) d t .
$$

If the time-scale of the GRB prompt emission and its shape is more less the same for any burst then $E_{\mathrm{p}}\left(\propto k T_{\mathrm{e}}\right)$ is $E_{\mathrm{iso}}^{1 / 2}$ which is precisely seen in the Amati relation Amati et al. (2002) and Amati (2006)]. The observable scattering of points in the Amati relation along correlation $E_{p} \propto E_{\text {iso }}^{1 / 2}$ can be caused by the spread of the parameter values that characterize the outflow evolution of each GRB like the bulk velocity $V_{b}$ and the spectral index of the relativistic phase $\alpha_{b}$ (see Eq. 18 and Fig. 7). Another reason for the spread in the Amati relation is the different temperature of the seed (soft) photons, which is related to the emission of the star itself.

\section{Temporal variability}

One of the most established observational results of GRBs since the beginning is the high temporal variability of their light curves (Meegan et al. 1992), which can extend down to $10^{-3} \mathrm{~s}$. More recently, in addition to the short time variability, also a slow time variability, with time scales from several seconds to $\sim 100 \mathrm{~s}$ has been discovered, with the GRB prompt light curves being the superposition of both components (e.g., Vetere et al. 2006; Gao et al. 2012). In particular, Vetere et al. (2006), by performing the systematic analysis of a sample of GRBs detected with the WFCs aboard BeppoSAX also showed that the slow component is more pronounced at lower energies $(2-10 \mathrm{keV})$ than at high energies (10-26 $\mathrm{keV})$. Accumulating evidence from the Swift era suggests that at least the slow component of the GRB variability is closely related to the central engine (Liang et al. 2006), which was confirmed also by other investigations (Gao et al. 2012).

Now we demonstrate that, in our scenario, the slow component with temporal variability of order 1-100 seconds is related to the Compton cloud while the fast variability component with scale of $\ll 1 \mathrm{~s}$ is presumably related to the relativistic outflow (jet). In order to estimate the time scale of emergent radiation from the hot Compton cloud one should take a difference of the arrival time from the outskirt $t_{\text {out }}$ and center of Compton cloud $t_{\mathrm{c}}$ (see Fig. 1). Namely

$$
\Delta t=t_{\mathrm{out}}-t_{\mathrm{c}}
$$

where $t_{\text {out }}=\rho_{\text {out }} / c, t_{\mathrm{c}}=d / c$ and $d, \rho_{\text {out }}$ are the distances from the observer to the center and outskirt of the hot spot respectively (see Fig. 1). Thus

$$
\rho_{\text {out }}=\sqrt{R^{2} \sin ^{2} \varphi+[d+(R-R \cos \varphi)]^{2}}
$$


where $R$ is the radius of the star and $\varphi$ is angle between the vector directed from the center of the star to outskirt point and vector directed towards the observer. Then for $R \ll d$ we find that

$$
\Delta t=t_{\mathrm{out}}-t_{\mathrm{c}} \approx R(1-\cos \varphi) / c
$$

and for $\varphi \ll \pi / 2$ we obtain

$$
\Delta t \approx \varphi^{2} R / 2 c
$$

For example if $\varphi<0.1$ (or $\varphi<5^{\circ}$ in degrees) then the variability time scale of the emergent radiation observed from the Compton cloud should be

$$
\Delta t<15\left(R / 10^{14} \mathrm{~cm}\right) \mathrm{s} .
$$

which is a typical long time scale of the GRB prompt emission (see above).

On the other hand short time scale variability $(t \ll 1 \mathrm{~s})$ is presumably related to relativistic outflow (see section 2.3 and below). Variations of $\mathrm{X}$-ray $-\gamma$ radiation in the outflow in time scale $T$ detected by the Earth observer as

$$
T_{0}=T\left[1-\left(V_{b} / c\right) \cos \theta\right]
$$

where $V_{b}$ is the outflow velocity in the relativistic phase and $\theta$ is angle between the direction of the outflow motion and the direction to the Earth observer [see Rybicki \& Lightman $(1979)]$. If the observer sees the outflow motion at $\theta \ll 1$ then the time scale is very short

$$
T_{0}=T /\left(2 \Gamma_{L}^{2}\right)
$$

where $\Gamma_{L}$ is the Lorentz factor of the outflow(jet). It means that the short variability time scales should be only seen when the jet is directed towards the observer. The short temporal variability seen in GRB of order of $10^{-3}-10^{-1} \mathrm{~s}$ can be considered a signature of the outflow for which time scales of $\mathrm{X}$-ray $-\gamma$ variations of order of seconds are seen by the Earth observer as $10^{-3}-10^{-1}$-s variations. In other words the long time scales of order $10-100 \mathrm{~s}$ (intrinsic variabilities of X-ray radiation in the hot spot region) are related to the Comptonized radiation of the hot spot in the subrelativistic phase while the short time scales of order $10^{-3}-10^{-1} \mathrm{~s}$, as seen by the Earth observer, are originated mainly in the relativistic outflow phase similar to that in the standard fireball scenario (e.g. Mészáros 2006).

Using the emergent spectra itself one cannot distinguish the contribution of Compton cloud and the outflow in the spectrum below the peak energy $E_{\mathrm{p}}$ because the Comptonization spectrum is wider than the upscattering Green function of the outflow, at least for $\alpha_{b}>1.5$ (see section 2.3). The short and long time variabilities should be seen in the same energy range at $E_{\sim}^{<} E_{\mathrm{p}}$ but the short one should be mostly observed alone at high energies (above $\left.E_{\mathrm{p}}\right)$ where the pure extended power-law component is detected. 


\section{Discussion}

We have developed a Comptonization model aimed to describe the prompt spectral emission of GRBs over a broad energy range. The assumption is the presence of two ingredients: a thermal bath of soft X-ray photons and the existence of a hot plasma outflow which should be formed as a consequence of the explosion of a star. Both ingredients were also assumed by Lazzati et al. (2000) for their Compton-drag model to interpret the prompt emission from GRBs associated with supernovae. In our model, the hot plasma outflow has a bulk velocity which is initially subrelativistic $\left(V_{\mathrm{b}} / c \lesssim 0.1-0.2\right)$ and later it becomes relativistic outside the star photospheric radius. Note that a model consisting of an early subrelativistic followed by a relativistic phase has been proposed by (Lyutikov \& Blandford 2003) in the context of GRBs originated by electromagnetic outflow.

Our model consists of two parts: the first one describes the spectra up to the peak energy in the $\mathrm{EF}(\mathrm{E})$ diagram and is obtained from the solution of the Fokker-Planck expansion of the radiative transfer equation which takes into account multiple scattering of a BB-like seed photon population off a hot and thick electron plasma [Compton cloud $(\mathrm{CC})$ ]. The relatively soft $\mathrm{BB}$ population $\left(k T_{\mathrm{bb}} \sim 1 \mathrm{keV}\right)$ presumably originates in the relatively cold star photosphere and illuminates CC (see Fig. 1). The spectral output of this early phase is characteristic of saturated Comptonization, but the shape slightly deviates from a pure Wien law because of the modification due to outflow motion (see Figs. 2 and 3). In this case, the peak of the spectrum in the $\mathrm{EF}(\mathrm{E})$ diagram falls slightly below $E_{\mathrm{p}} \sim 4 k T_{\mathrm{e}}$, with respect to that for the standard Comptonization where the bulk outflow velocity $V_{b}=0$.

The second part of our model describes the prompt emission spectra beyond the peak energy $E_{p}$. This was obtained by an assumption that, beyond the photosphere, the outflow velocity becomes relativistic and provides a further upscattering of the already Comptonized spectrum. We discuss now some relevant points related to our model.

It is important to estimate the thermalization optical depth $\tau_{\text {eff }}$, which also appears in the definition of $\delta$ (see Eq. 9). This quantity indeed is not an explicit parameter of the Comptonization equation (8). Using the best-fit values of $k T_{\mathrm{e}}$ and $\delta$ for GRB990705 (with $\left.\beta_{0} \lesssim 0.2\right)$ we find $\tau_{\text {eff }} \sim 2$.

From the continuity equation in spherical symmetry we find

$$
N_{\mathrm{e}} \approx 4 \times 10^{14} \frac{\left(\dot{m} / 10^{-5}\right)}{\left(\xi_{\Omega} / 0.5\right) r_{14}^{2}\left(\beta_{0} / 0.1\right)} \mathrm{cm}^{-3},
$$

where $\dot{m} \equiv \dot{M} / \dot{M}_{\odot}\left(\dot{M}_{\odot} \approx 10^{33} \mathrm{~g} \mathrm{~s}^{-1}\right), r_{14} \equiv R /\left(10^{14} \mathrm{~cm}\right), \xi_{\Omega}=2 \pi(1-\cos \theta)$ and $\theta$ is the half opening angle of the subrelativistic outflow. 
Then optical depth of corona located on the top of the star is

$$
\tau_{0}=N_{\mathrm{e}} \sigma_{\mathrm{T}} H \sim 7 \frac{\left(\dot{m} / 10^{-5}\right)\left(H / 3 \times 10^{10} \mathrm{~cm}\right)}{\left(\xi_{\Omega} / 0.5\right) r_{14}^{2}\left(\beta_{0} / 0.1\right)},
$$

where $H$ is the thickness of the hot corona.

\subsection{The resulting GRB luminosity}

The total luminosity of the observable GRB emission can be estimated analytically using the same procedure defined in ST80 and CT95. Let us first define the BB luminosity illuminating the hot Compton cloud as

$$
L_{b b, i l}=5 \times 10^{46}\left(\xi_{\Omega} / 0.5\right)\left(k T_{\mathrm{bb}} / 0.1 \mathrm{keV}\right)^{4} r_{14}^{2} \mathrm{erg} \mathrm{s}^{-1}
$$

Using the formula for the enhancement factor $\eta_{\text {comp }}$ (see equation 20) for the case of saturated Comptonization $(\alpha \ll 1)$ we obtain

$$
L_{\text {grb }}^{\text {comp }}=\eta_{\text {comp }} L_{b b, i l} \sim 5 \times 10^{49}\left(T_{100}^{(\mathrm{e})} / T_{0.1}^{(\mathrm{bb})}\right)\left(\xi_{\Omega} / 0.5\right)\left(T_{0.1}^{(\mathrm{bb})}\right)^{4} r_{14}^{2} \operatorname{erg~s}^{-1},
$$

where $T_{100}^{(\mathrm{e})} \equiv k T_{\mathrm{e}} /(100 \mathrm{keV})$ and $T_{0.1}^{(\mathrm{bb})} \equiv k T_{\mathrm{bb}} /(0.1 \mathrm{keV})$, respectively.

If the high-energy power law index of the hard component, which is presumably formed due to the inverse Compton effect, is $\alpha_{\mathrm{b}} \geq 2$ then the related enhancement factor $\eta_{\text {rel }}$

$$
\eta_{\mathrm{rel}}=\frac{\alpha_{\mathrm{b}}\left(\alpha_{\mathrm{b}}+3\right)}{\left(\alpha_{\mathrm{b}}-1\right)\left(\alpha_{\mathrm{b}}+4\right)}
$$

is order of 1 . Thus the resulting enhancement factor $\eta_{\text {res }} \sim \eta_{\text {comp }}$ and the GRB resulting luminosity $L_{\text {grb }}=\eta_{\text {rel }} L_{\text {grb }}^{\text {comp }} \sim L_{\text {grb }}^{\text {comp }}$.

\subsection{On the origin of the high-energy power-law component}

As we already outlined in Section 2.3, subrelativistic bulk motion Comptonization is characterized by a rollover feature around an energy which is of the order of the electron temperature, slightly modified by the presence of matter velocity field. In any case, this process cannot give rise to any $\mathrm{PL}$ component extending up to $\mathrm{GeV}$ as observed in GRBs (e.g., Abdo et al. 1992). Actually a second component must be included in the X/ $\gamma$-ray spectral model. If the subrelativistic phase Comptonization spectrum and the high-energy 
PL have different origin, then in terms of spectral modeling the latter could be described by simply adding a PL spectral feature. However, a physically unconnected origin of the two components seems unlikely, given that all the GRB spectra show a smooth joining of the low-energy and high-energy features around the peak energy, and the GeV emission for most Fermi GRBs is consistent with the extrapolation of the MeV component (Zhang et al. 2011, hereafter Z11).

Here, we suggest this physical link in terms of the solution of Equation (19) where the bulk Comptonization spectrum of the subrelativistic phase (Eq. 8) is convolved with a broken-PL GF (Eq. 18) leading to the emergent broad-band spectrum (see Figs. 66-7).

The critical point to be addressed is of course the nature of the upscattering process that gives rise to the suggested GF (Eq. 18). The apparently non-thermal origin of the highenergy GRB component leads to include mechanisms of particle non-thermal acceleration in shock regions. The standard fireball model actually allows the possibility to consider shocks as possible sites for particle acceleration, which eventually lead to non-thermal distributions $N_{\mathrm{e}}(E) \propto E^{-\mathrm{p}}$. In this context, the high-energy PL would be produced through synchrotron and/or inverse processes by this non-thermal particle bath. An intermediate case where the electron population has a Maxwellian distribution plus a supra-thermal tail was also considered by Tavani (1996).

One of the key assumptions of the fireball scenario is that the GRB outflow is highly relativistic since the beginning of the event, with $\Gamma \gtrsim 100$. In our model, however, the first phase of the GRB phenomenon is subrelativistic. How to conciliate this significant difference? Is really an unavoidable requirement that the photosphere is ultra-relativistic? Let us consider this possible scenario: when the top bounce of the hot shell emerges from the star photosphere with bulk velocity $V_{\mathrm{b}} / c \lesssim 0.1$, the underlying star photospheric luminosity is huge ( $L_{*} \gtrsim 10^{10} L_{\text {Edd }}$ ), and it illuminates the shell itself, which subtends a solid angle $\Omega / 4 \pi<1$. This particular configuration (hot plasma, anisotropic high radiation pressure) could be suitable to give rise to an efficient plasma acceleration through the so-called "Compton-rocket effect" (Cheng \& Odell 1981). For instance, in the case of an illuminating point source, the ratio of the radiative forces acting on a hot and cold plasma, respectively, is $f_{\text {hot }} / f_{\text {cold }}=2 / 3<(\gamma \beta)^{2}>$ (Odell 1981), where $\left\langle(\gamma \beta)^{2}>\right.$ is the quadratic average momentum of a Maxwellian electron distribution. Numerical simulations by Renaud \& Henri (2000) show that electron-positron jet populations with non-thermal distribution illuminated by an underlying accretion disk can be accelerated up to Lorentz factors of several tens.

It is beyond the scope of our paper to investigate quantitatively the hydrodynamical configuration leading to plasma acceleration under the underlying star radiation field. Here we just claim a qualitative scenario where the accelerated plasma may interact with the 
interstellar medium and/or star wind producing shocks with associated particle non-thermal populations. The high-energy photons of the subrelativistic phase then interact with these electrons via inverse Compton effect (Fig. 11) and the GF of such a process in given by equation (18). Note that in this case, the radiative production mechanism is not qualitatively different from the standard fireball model. No matter whether synchrotron or inverse Compton dominate the spectral formation, what is important is the total electron Lorentz factor, $\Gamma_{\text {tot }} \sim \Gamma_{\mathrm{sh}} \gamma_{e}$, where $\Gamma_{\mathrm{sh}}$ and $\gamma_{e}$ are the Lorentz factors of the expanding shell and of the electron in the shell frame, respectively. A quantitative treatment of the radiation transfer problem is needed in this case.

Other models have been proposed to explain the GRB prompt emission in the framework

of inverse Compton processes (see reviews by Mészáros 2006; Gehrels et al. 2012). The key difference between our model and the other photospheric or synchrotron scenarios is that in our model case the energy peak of the $E F(E)$ diagram is independent of any Lorentz $\Gamma_{L}$-factor associated to relativistic expansion. The main parameter driving the peak position (the electron temperature $k T_{\mathrm{e}}$ ) is essentially the same in the fluid and observer frame (apart from cosmological redshift effects), unlike that suggested by Ghirlanda et al. (2012) who claim that the extension of $E_{p}$ energy of the $E F(E)$-diagram to a few $\mathrm{MeV}$ is mostly due to distribution of Lorentz factors in the relativistic shell (or jet).

\subsection{The scenario in the light of the Fermi results}

The thermal nature of GRB spectra is still subject to debate. Blackbody components are predicted in various models (Mészáros \& Rees 2000; Peer et al. 2006; Thompson et al. 2007; Toma et al. 2011) and actually they have been found in time resolved GRB spectra using the BeppoSAX Gamma-Ray Burst Monitor and Wide Field Camera 2 (Frontera et al. 2001) and BATSE instruments (Ghirlanda et al. 2003; Ryde 2004, 2005, RP09). However, from the recent broad band observations with the Fermi GRBM plus LAT instruments it is not so clear whether the existence of a thermal component is a general property of GRBs. Indeed, on the basis of the systematic and detailed time-integrated and time-resolved analysis of a sample of 17 GRB data observed with GBM and LAT, Z11 found that most of the GRBs in this sample could be fitted with a Band function over the whole Fermi energy range. Only in two cases (GRB 090902B and possibly GRB 090510) the spectrum was peculiar, in that it could be described by a BB+PL model, similarly to what reported in RP09. The paradigm of Z11 is that the Band-only spectra are better consistent with a Poynting-dominated rather than baryon-dominated flow, thus discarding in most (albeit not all) cases a photospheric scenario. Against a pure photospheric emission the authors argue that the extension is up 
to $\mathrm{GeV}$ energies.

The preponderance of the non-detection of high temperature BB-like features in the Fermi GRB spectra as reported in Z11 is not, in our opinion, necessarily in contrast with our photospheric scenario. Indeed, in our proposed model the seed thermal photons and the electrons are decoupled and the low-energy slope of the emerging spectrum (up to the peak energy $E_{\mathrm{p}}$ ) is mostly dictated by the optical depth and plasma temperature of the hot corona and slightly modified by subrelativistic outflow velocity $V_{\mathrm{b}} \ll c$ (see Figs. 3 and 6 ). The Band function itself can thus be yet the result of photospheric Comptonization process, but with a different configuration of the radiation field and electron plasma (decoupled) with respect to the standard scenario (where both are coupled). It is also worth noting that the above-mentioned BB features detected in BASTE and Fermi spectra have characteristic observer-frame temperatures of orders of hundreds $\mathrm{keV}$, and thus must not be confused with the cool BB seed photons $(\stackrel{\sim}{\sim} \mathrm{keV})$ of our model. More specifically, we identify these hot BB component with the optically thick subrelativistically expanding plasma of the earlier phase in our scenario.

Actually, an important theoretical prediction of our model, to be tested with observations, is the spectral steepening at low-energies in comparison with the Band function (see Fig. 7). The change of slope is predicted to occur below $\sim 4 k T_{\mathrm{bb}} \mathrm{keV}$ in the $\mathrm{EF}(\mathrm{E})$ diagram, where $k T_{\mathrm{bb}}$ is the seed photon $\mathrm{BB}$ temperature, and it does represent the low-energy tail (Rayleigh-Jeans law) of the input BB. Thus a spectral slope $F(E) \propto E^{2}$ should be a universal GRB observational feature at soft X-ray energies (see also Fig. 6). Unfortunately, this issue cannot be solved by Swift or Fermi as their energy thresholds are about $15 \mathrm{keV}$ or $8 \mathrm{keV}$, respectively. The BeppoSAX GRBs observed with both GRBM and WFCs are more suitable to be used to test our model, even if the lower threshold of the passband (2 keV) is nearby the limit of the energy range foreseen for the seed photons. A test of our model has been performed using the BeppoSAX data(Frontera et al. 2012, in preparation).

\section{Conclusions}

We have developed a spectral model aimed to describe the broadband prompt emission of GRBs. We propose that the spectral emission during the prompt phase, phenomenologically modeled by the Band function, is the result of an earlier phase where soft BB-like photons are Comptonized by an optically thick and hot electron shell $\left(T_{\mathrm{e}} \sim 10^{9} \mathrm{~K}\right)$, something like a Compton cloud sub-relativistically moving outwards the star surface. On the other hand in the relativistic phase, these Comptonized photons are subjected to a second upscattering process which can be mathematically described by a broken power-law Green function whose 
spectral index models the high-energy slope of the Band function. An important prediction of our proposed model is that the peak energy in the $\mathrm{EF}(\mathrm{E})$ diagram originated in the early subrelativistic phase (see Fig. 1) is directly related to the plasma temperature of the hot plasma $T_{e}$. We demonstrate that the resulting luminosity of $X / \gamma$-rays luminosity of $G R B$ $L_{\mathrm{grb}}$ is proportional to $\left(k T_{\mathrm{e}}\right)^{2}$ (see Eq. 25).

In fact , $L_{\text {grb }}$ is a product of the Comptonization enhancement factor $\eta_{\text {comp }}$ and luminosity of the soft blackbody photons $L_{b b, i l}$ but in the case of the saturated Comptonization, when $\alpha \ll 1, \eta_{\text {comp }} \propto k T_{\mathrm{e}}$ (see Eq. 20) but $L_{\mathrm{bb} \text {,il }}$ (or $L_{\text {soft }}$ ) is also proportional to $k T_{\mathrm{e}}$ because the surface area of Compton cloud illuminated by the soft photons is proportional to $k T_{\mathrm{e}}$ (see Eq. 24). Thus we claim that the model dependence of $L_{\mathrm{grb}} \propto\left(k T_{\mathrm{e}}\right)^{2}$ on the hot plasma temperature $T_{\mathrm{e}}$ explains the observed Amati relation in which $E_{i s o}=\int L_{\mathrm{grb}}(t) d t \propto E_{\mathrm{p}}^{2}$. It is worth noting that the peak energy $E_{\mathrm{p}}$ of the emergent Wien spectrum should be equal to $3 k T_{\mathrm{e}}$.

Our model also explains both the observed long and short temporal variability during GRB prompt emission phase. The former one is related to time scale of the radiation formed in the sub-relativistically moving Compton cloud while the short time scale is related to time scale of radiation formed in the sub-relativistically moving Compton hot spot while the short time scale is related to the time scale of radiation coming to the Earth observer from the relativistically driven outflow.

We do not quantitatively investigate the physical conditions underlying the origin of the high-energy component. Different scenarios, not necessarily excluding each other, can be operating such as a second inverse Compton process off non-thermal electrons accelerated by super Eddington radiation pressure (for which $L \gg L_{E d d}$ ) as the hot shell moves out of the star. Self-synchrotron Compton or direct synchrotron effects can not be excluded. An important prediction of our model is that the peak energy in the $\mathrm{EF}(\mathrm{E})$ diagram originates in the early sub-relativistic phase and is proportional to plasma temperature $k T_{\mathrm{e}}$ and the resulting luminosity $L_{\text {grb }}$ is proportional to $\left(k T_{\mathrm{e}}\right)^{2}$. This dependence is the same, after cosmological corrections, in the source and observer frame. In fact, no fine tuning related to some Lorentz $\Gamma$-factor of relativistic expansion is required. We claim that this result of our model naturally explains the physical origin of the Amati relation.

A systematic application of our model to a sample of time-resolved GRB prompt spectra of the BeppoSAX archive by Frontera et al. (2012, to be submitted) proves that in all the cases the $\chi^{2}$-values are equal or better than those derived with the Band function. To our knowledge, this is the first time that a model for the GRB prompt emission other than the Band function has been developed under the X-ray spectral fitting package XSPEC. 
Acknowledgements The authors acknowledge very productive discussions with Davide Lazzati, Pawan Kumar and Alexandre Chekhtman which strongly improved the quality of our paper. Important suggestions have been given by the anonymous referee, which have allowed us to better clarify the main topics of our model.

\section{A. Numerical solution of Fokker-Planck radiative transfer equation using finite differences}

We consider the Fokker-Planck expansion of the radiative transfer equation for the zeromoment occupation number $\mathrm{N}(\mathrm{x})$, in the presence of subrelativistic bulk motion (see Eq. 8):

$$
f_{\mathrm{b}} x^{2} \frac{d^{2} N(x)}{d^{2} x}+\left(x^{2}+4 f_{\mathrm{b}} x+\delta x\right) \frac{d N(x)}{d x}+(4 x-\gamma) N(x)=-\frac{\varphi(x)}{x^{3}}
$$

where $x \equiv E / k T_{\mathrm{e}}, \Theta \equiv k T_{\mathrm{e}} / m_{\mathrm{e}} c^{2}, f_{\mathrm{b}}=1+3 \beta_{0}^{2} / \Theta$, while $\gamma$ and $\delta$ are defined in Sect. 2.2, For $\varphi(x)$ we assume a BB spectrum (see Eq. B1).

In terms of the zero-moment intensity $J(x)=N(x) x^{3}$ the equation becomes

$$
f x^{2} \frac{d^{2} J(x)}{d^{2} x}+\left(x^{2}-2 f x+\delta x\right) \frac{d J(x)}{d x}+(x-\gamma-3 \delta) J(x)=-\varphi(x) .
$$

In order to provide logarithmic binning of the a dimensional energy $x$, we introduce the new variable $y$ such that $x=e^{y}$, thus obtaining the new equation in terms of this variable

$$
f \frac{d^{2} J(y)}{d^{2} y}+\left(e^{y}-3 f+\delta\right) \frac{d J(y)}{d y}+\left(e^{y}-\gamma-3 \delta\right) J(y)=-\varphi(y) .
$$

For numerical integration, we divide the domain of $y$ from $y_{\min }$ to $y_{\max }$ into $\mathrm{N}$ equallystepped sizes $h=\left(y_{\max }-y_{\min }\right) / N$, so that equation can be presented, using finite differences, as

$$
p_{\mathrm{n}} \frac{J_{\mathrm{n}+1}-2 J_{\mathrm{n}}+J_{\mathrm{n}-1}}{h^{2}}+g_{\mathrm{n}} \frac{J_{\mathrm{n}+1}-J_{\mathrm{n}}}{h}+r_{\mathrm{n}} J_{n}=-\varphi_{\mathrm{n}}
$$

where

$$
p_{\mathrm{n}}=f, \quad g_{\mathrm{n}}=\left(e^{y_{\mathrm{n}}}-3 f-\delta\right) \quad \text { and } \quad r_{\mathrm{n}}=\left(e^{y_{\mathrm{n}}}+3 \delta-\gamma\right) .
$$

The two boundary conditions for the intensity $\mathrm{J}(\mathrm{x})$ are

$$
J[0]=0, \quad J[N]=0,
$$

resembling the physical conditions $J(x)=0$ for $x \rightarrow 0$ and $x \rightarrow \infty$. 
Collecting the terms with the same index in equation (A4) and using the boundary conditions (A6), we may rewrite the equation as

$$
a_{\mathrm{n}} J_{\mathrm{n}-1}+b_{\mathrm{n}} J_{n}+c_{\mathrm{n}} J_{\mathrm{n}+1}=g_{\mathrm{n}},
$$

with coefficients $a_{\mathrm{n}}, b_{\mathrm{n}}, c_{\mathrm{n}}$ defined as

$$
\left\{\begin{array}{l}
a_{0}=0, \quad b_{0}=-1, \quad c_{0}=0, \quad \varphi_{0}=0, \quad n=0 \\
a_{\mathrm{n}}=\frac{p_{\mathrm{n}}}{h^{2}}, \quad b_{\mathrm{n}}=-\left(\frac{2 p_{\mathrm{n}}}{h^{2}}+\frac{g_{\mathrm{n}}}{h}-r_{\mathrm{n}}\right), \quad c_{\mathrm{n}}=\frac{p_{\mathrm{n}}}{h^{2}}+\frac{g_{\mathrm{n}}}{h}, \quad \varphi_{n}=\varphi\left(t_{n}\right), \quad \text { for } n=1, \ldots N-1,(\mathrm{~A} 8) \\
a_{\mathrm{N}}=0, \quad b_{\mathrm{N}}=1, \quad c_{\mathrm{N}}=0, \quad \varphi_{\mathrm{N}}=0, \quad n=N .
\end{array}\right.
$$

For $n=0$ Equation (A7) can be presented as

$$
J_{0}=L_{0} J_{1}+K_{0}, \quad \text { where } L_{0}=-c_{0} / b_{0}, \text { and } K_{0}=0 \text {. }
$$

Excluding $J_{0}$ from equation (A7) for $n=1$, we obtain

$$
\left(a_{1} L_{0}+b_{1}\right) J_{1}+c_{1} J_{2}=g_{1}-a_{1} K_{0} .
$$

Because $a_{1} L_{0}+b_{1} \neq 0$ we also obtain that

$$
J_{1}=L_{1} J_{2}+K_{1}
$$

where we have introduced new coefficients

$$
L_{1}=-\frac{c_{1}}{a_{1} L_{0}+b_{1}}, \text { and } K_{1}=\frac{g_{1}-a_{1} K_{0}}{a_{1} L_{0}+b_{1}} .
$$

Continuing the process of exclusion of $J_{n}$ we obtain in general

$$
J_{\mathrm{n}}=L_{\mathrm{n}} J_{\mathrm{n}+1}+K_{\mathrm{n}}
$$

where $L_{\mathrm{n}}=-\frac{c_{\mathrm{n}}}{a_{\mathrm{n}} L_{\mathrm{n}-1}+b_{\mathrm{n}}}$, and $K_{\mathrm{n}}=\frac{g_{\mathrm{n}}-a_{\mathrm{n}} K_{\mathrm{n}-1}}{a_{\mathrm{n}} L_{\mathrm{n}-1}+b_{\mathrm{n}}}$. 
Now, using the right boundary condition $\mathrm{J}[\mathrm{N}]=0$ and relation $(\underline{\mathrm{A} 13})$ it is possible to find

$$
J_{\mathrm{N}-1}=L_{\mathrm{N}-1} J_{\mathrm{N}}+K_{\mathrm{N}-1}
$$

and so on until all $J_{\mathrm{N}-2}, J_{\mathrm{N}-3}, \ldots J_{3}, J_{2}, J_{1}, J_{0}$ are calculated. This algorithm was first introduced by Israel Gelfand is called by the sweep (progonka) method.

In order to reproduce the unbroken PL Component of the high-energy spectrum, we solved Equation (19) for the zero moment intensity, replacing $\varphi(x)$ with $F_{\mathrm{tb}}(x)$ which gives

$$
x^{2} \frac{d^{2} J(x)}{d^{2} x}-2 x \frac{d J(x)}{d x}-\alpha_{\mathrm{b}}\left(\alpha_{\mathrm{b}}+3\right) J(x)=-F_{\mathrm{tb}}(x) .
$$

As we have already shown in Sect. 2.3, this is equivalent to calculation of the convolution integral in Equation (11) with the GF given by formula (18) and $\varphi(x)=F_{\mathrm{tb}}(x)$. The procedure for the numerical solution of equation (A16) is identical to that used for solving Equation (A1) but just changing the expression for the cofficients $a_{\mathrm{n}}, b_{\mathrm{n}}$ and $c_{\mathrm{n}}$.

\section{B. Development of a spectral model for XSPEC}

We develop a numerical model for the X-ray spectral fitting package XSPEC. The model can be actually divided into two parts, one strictly physical, in terms of the parameter setting, and the second one which is more phenomenological.

\section{Step 1: Comptonization in subrelativistic outflow}

The first part of the model computes the spectrum obtained as a result of Comptonization of a BB-like seed photon population off a hot electron corona which is subrelativistically expanding, according to equation (1). For practical purposes, actually we solved equation (8), which is obtained from the variable separation method, with the bulk parameter $\delta$ defined in Eq. (9).

The input seed spectrum is given by the Planck's law

$$
\varphi(x)=N_{\mathrm{bb}}\left(k T_{\mathrm{e}}\right)^{3} \frac{x^{3}}{e^{\left(k T_{\mathrm{e}} / k T_{\mathrm{bb}}\right) x}-1},
$$

where $x \equiv E / k T_{\mathrm{e}}$, while $k T_{\mathrm{bb}}$ and $k T_{\mathrm{e}}$ are the $\mathrm{BB}$ and electron temperatures, respectively.

The normalization constant $N_{\mathrm{bb}} \equiv R_{9}^{2} / D_{\mathrm{Mpc}}^{2}$, where $R_{9}$ is the BB apparent photospheric radius in units of $10^{9} \mathrm{~cm}$ and $D_{\mathrm{Mpc}}$ is the source distance in $\mathrm{Mpc}$, respectively. Note that 
this is the source term appearing in the right-hand side of equation (8). We numerically solved the latter equation using a fast finite-difference method (see Appendix A) rather then performing a convolution with GF defined in Eq. (11). The free-parameters of the model at this step are the $\mathrm{BB}$ temperature and normalization ( $k T_{\mathrm{bb}}$ and $N_{\mathrm{bb}}$, respectively), the electron plasma temperature $k T_{\mathrm{e}}$, the bulk parameter $\delta$, the $\gamma$-parameter (which, in fact, is the inverse of the Comptonization parameter $Y$ ) and the second power of the (constant) outflow velocity $\beta_{0}^{2}$.

We note that the outflow velocity $\beta_{0}$ appears in Equation (8) both explicitly in the term $f_{\mathrm{b}}=1+\beta_{0}^{2} /(3 \Theta)$ and implicitly in the definition of the $\delta$-parameter (see Eq. 9). The linear dependence on $\beta_{0}$ via the bulk parameter $\delta$ is related to the effect of Fermi first-order photon down (up)-scattering and the net result is photon red-shift or blue-shift, depending on the sign of $\nabla \cdot \mathbf{V}_{\mathrm{b}}$, while the quadratic dependence of $\beta_{0}$ in $f_{\mathrm{b}}$ provides the second-order Fermi process and represents an up-scattering contribution (when averaged over angles) term in addition to that coming from the electron thermal velocity component. By setting $\beta_{0}^{2} \ll 3 \Theta$, it is possible to find the analytical solution for the emerging spectrum $F_{\mathrm{tb}}(x)$ using convolution (11) and the analytical presentation of the GF (see Eq. 12). A comparison between the numerical and analytical solution in this limit has shown an excellent agreement between the two cases.

\section{Step 2: high-energy powerlaw component}

We should point out that in principle a general broken-PL GF is described by two indexes for $E<E_{0}$ and $E>E_{0}$, while in the GF of equation (18) they both depend on $\alpha_{\mathrm{b}}$. However, for our purposes, it is important that the red wing of the GF, $\propto E^{\alpha_{\mathrm{b}}+3}$, is significantly steeper than the blue wing $\propto E^{-\alpha_{\mathrm{b}}}$. Actually, it is this up-scattering term of the GF which dominates the convolution process. We show an example of spectrum obtained from the solution of Eqs. (8) and (19) compared with the Band function in Figure 7. It is evident their similarity, but we also draw the attention of the reader to the change in the slope at low energy of our model with respect to the Band function. The low-energy steepening occurs at $E \lesssim 4 k T_{\mathrm{bb}}$ in a $\mathrm{EF}(\mathrm{E})$ diagram, where $k T_{\mathrm{bb}}$ is the temperature of the seed $\mathrm{BB}$ photons, and it is related to the Rayleigh-Jeans part of the Planckian distribution, where $F(E) \propto E^{2}$. For the case reported in Fig. 7 we assumed $k T_{\mathrm{bb}}=1 \mathrm{keV}$, so that the steepening occurs below $4 \mathrm{keV}$. We subsequently implemented our model in the XSPEC package and tested it on GRB990705 ( $\mathrm{z}=0.842$; Amati 2006), observed by the BeppoSAX/WFC+GRBM instruments. 


\section{REFERENCES}

Abdo, A. A. et al. 2009, Science, 323, 1688

Amati, L. 2006, MNRAS, 372, 233

Amati L., Frontera, F. \& Guidorzi, C. 2009, A\&A, 508, 173

Amati, L. et al. 2002, A\&A, 390, 81

Amati L. et al. 2008, MNRAS, 391, 577

Band D. et al. 1993, ApJ, 413, 281

Becker, P.A. \& Wolff, M.T. 2007, ApJ, 654, 435

Beloborodov, A.M. 2010, MNRAS, 407, 1033

Blandford R.D. \& Payne, D.G. 1981, MNRAS, 194, 1041 (BP81)

Bychkov, V., Popov, M.V., Oparin, A.M., Stenflo, L. \& Chechetkin, V.M. 2006, Astronomy Reports, 50, 298

Chakrabarti, S. K. \& Titarchuk, L. G. 1995, ApJ, 455, 623

Chardonnet, P., Chechetkin, V. \& Titarchuk, L. 2010, Ap\&SS, 325, 153

Cheng, A.Y.S. \& Odell, S.L. 1981, ApJ, 251, L49

Colpi, M. 1988, ApJ, 326, 223

Cowsik, R. \& Lee, M.A. 1982, Royal Society of London Proceedings Series A, 383, 409

Crider, A., Liang, E.P. \& Preece, R.D.1998, in American Institute of Physics Conference Series, Gamma-Ray Bursts, Vol. 428, 4th Hunstville Symposium, Eds. C.A.Meegan, R.D. Preece, \& T.M. Koshut, 428, 359-363

Crider, A. et al. 1997, ApJ, 479, L39

Farinelli, R., Titarchuk, L. Paizis, A. \& Frontera, F. 2008, ApJ, 680, 602, (F08)

Fiore, F., Guetta, D., Piranomonte, S., D’Elia, V., Antonelli, L.A. 2007, A\&A, 470, 515

Frontera, F. et al. 2000, ApJS, 127, 59

Frontera, F. et al. 2001, ApJ, 550, L47 
Fryxell, B. et al. 2000, ApJS, 131, 273

Gal-Yam, A. et al. 2009, Nature, 462, 624

Gao, H. Zhang, B.B. \& Zhang, B. 2012, ApJ, 748, 134

Gehrels, N., Ramirez-Ruiz, E. \& Fox, D.B. 2009, ARA\&A, 47, 567

Ghirlanda, G., Bosnjak, Z., Ghisellini, G., Tavecchio, F. \& Firmani, C. 2007, MNRAS, 379, 73

Ghirlanda, G., Celotti, A. \& Ghisellini, G. 2003, A\&A, 406, 879

Ghirlanda, G. et al. 2012, MNRAS, online Early, arXiv:1203.0003

Ghirlanda, G., Nava, L. \& Ghisellini, G. 2010, A\&A, 511, A43

Imshennik, V.S., Kal'Yanova, N.L., Koldoba, A.V. \& Chechetkin, V.M. 1999, Astronomy Letters, 25, 206

Jakobsson, P. et al. 2006, A\&A, 447, 897

Kelly, P.L., Kirshner, R.P. \& Pahre, M. 2008, ApJ, 687, 1201

Langer, N., Norman, C.A., de Koter, A., Vink, J.S., Cantiello, M. \& Yoon, S.-C. 2007, A\&A, 475, L19

Laurent, P. \& Titarchuk, L. 2007, ApJ, 656, 1056 (LT07)

Laurent, P. \& Titarchuk, L. 1999, ApJ, 511, 289

Lazzati, D., Ghisellini, G., Celotti, A. \& Rees, M.J. 2000, ApJ, 529, L17

Lazzati, D., Morsony, B.J. \& Begelman, M.C. 2009, ApJ, 700, L47

Liang, E. et al. 2006, ApJ, 646, 351

Lyutikov, M. \& Blandford, R. 2003, arXiv:astro-ph/03035410

Lyutikov, M., Pariev, V.I. \& Blandford, R. 2003, ApJ, 597, 998

Meegan, C.A. et al. 1992, Nature, 355, 143

Mészáros, P. 2006, Reports on Progress in Physics, 69, 2259

Mészáros, P. \& 2000, ApJ, 530, 292 
Odell, S.L. 1981, ApJ, 243, L147 Thompson07

Pe’er, A., Mészáros, P. \& Rees, M.J. 2006, ApJ, 642, 995

Piran, T. 1999, Phys. Rep., 314, 575

Raskin, C., Scannapieco, E., Rhoads, J. \& Della Valle, M. 2008, ApJ, 689, 358

Rees, M. \& Mészáros, P. 2005, ApJ, 628, 847

Renaud, N. \& Henri, G. 2000, Nuclear Physics B Proceedings Supplements, 80, C125

Rybicki, G.B. \& Lightman, A.P. 1979, Radiative Processes in Astrophysics, New York, Wiley-Interscience, $393 \mathrm{p}$.

Ryde, F. 2005, ApJ, 625, L95

Ryde, F. 2004, ApJ, 614, 827

Ryde, F. \& Pe'er, A. 2009, ApJ, 702, 1211

Sunyaev, R.A. \& Titarchuk, L.G. 1985, A\&A, 143, 374

Sunyaev, R.A. \& Titarchuk, L.G. 1985, A\&A, 86, 121 (ST80)

Tavani, M. 1996, ApJ, 466, 768

Thompson, C. 1994, MNRAS, 270, 480

Thompson, C., Mészáros, P. \& Rees, M.J. 2007, ApJ, 666, 1012

Titarchuk, L. Kazanas, D. \& Becker, P.A. 2003, ApJ, 598, 411

Titarchuk, L., Mastichiadis, A. \& Kylafis, N.D. 1997, ApJ, 487, 834 (TMK97)

Toma, K., Wu, X.-F. \& Mészáros, P. 2011, MNRAS, 415, 1663

Vetere, L., Massaro, E., Costa, E., Soffitta, P. \& Ventura, G. 2007, A\&A, 447, 499

Woosley, S.E. 2011, arXiv:1105.4193

Woosley, S.E., Blinnikov, S. \& Heger, A. 2007, Nature, 450, 390

Zhang, B.B. 2011, ApJ, 730, 141, (Z11)

Zhang, B.B. \& Yan, H. 2011, ApJ, 726, 90 


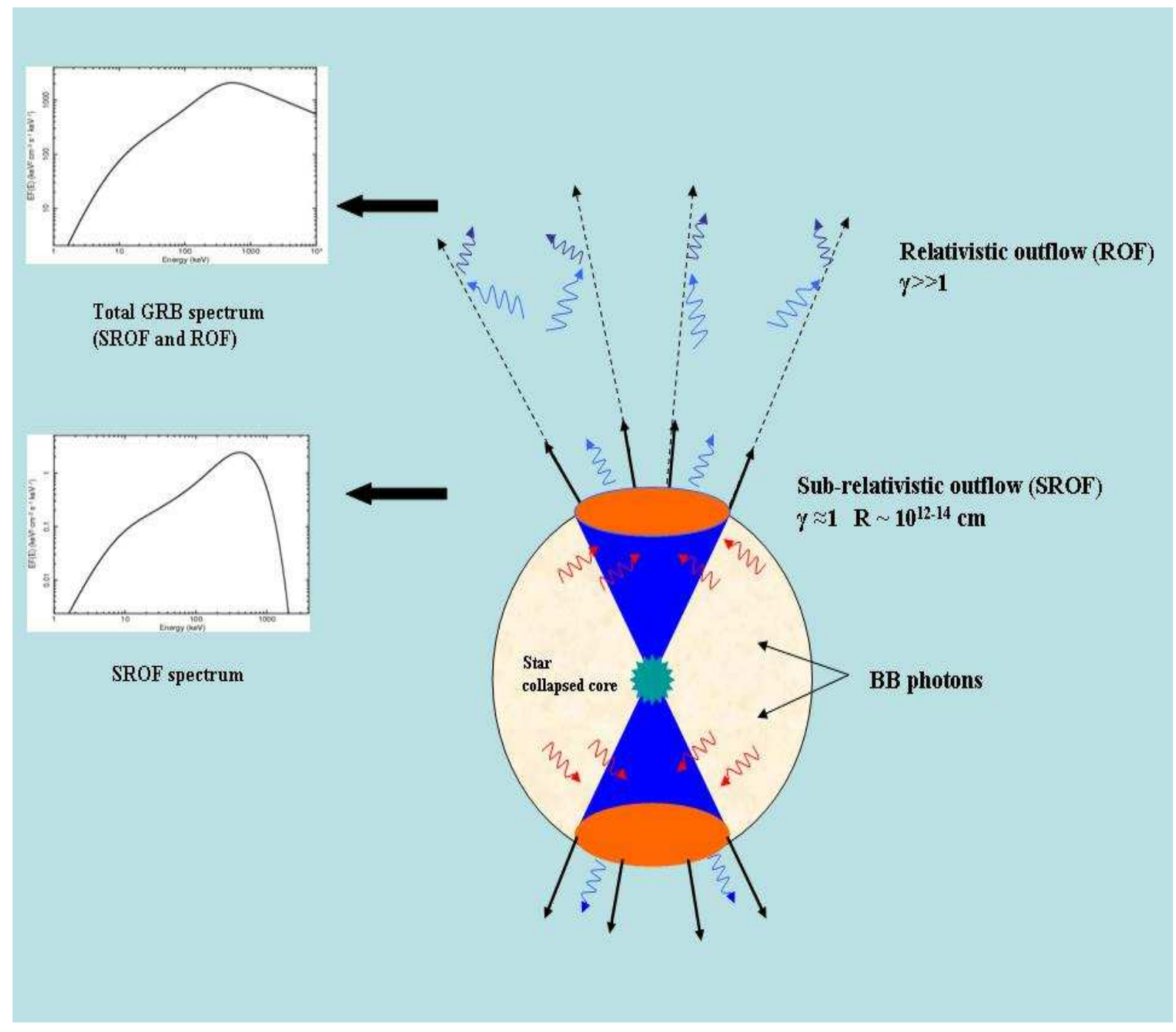

Fig. 1. - Schematic view of the explosion of a massive star and associated emergent spectral components. At the first stage, the spectrum is formed by Comptonization of blackbodylike seed photons (red arrows) placed at the bottom of a hot sub-relativistically outflow (SROF or Compton cloud) on the top of the star photosphere (orange region). The emergent Comptonization spectrum (bright-blue arrows) is obtained using the solution of Eq. (8). The Compton cloud can be accelerated by the underlying radiation pressure of the star photosphere leading to the relativistic outflow (ROF). Inverse Compton scattering of the Comptonized photons of the subrelativistic phase off non-thermal relativistic electrons of ROF can be the origin of the extended power-law observed above the peak in the $\mathrm{EF}(\mathrm{E})$ diagram (dark-blue arrows). 


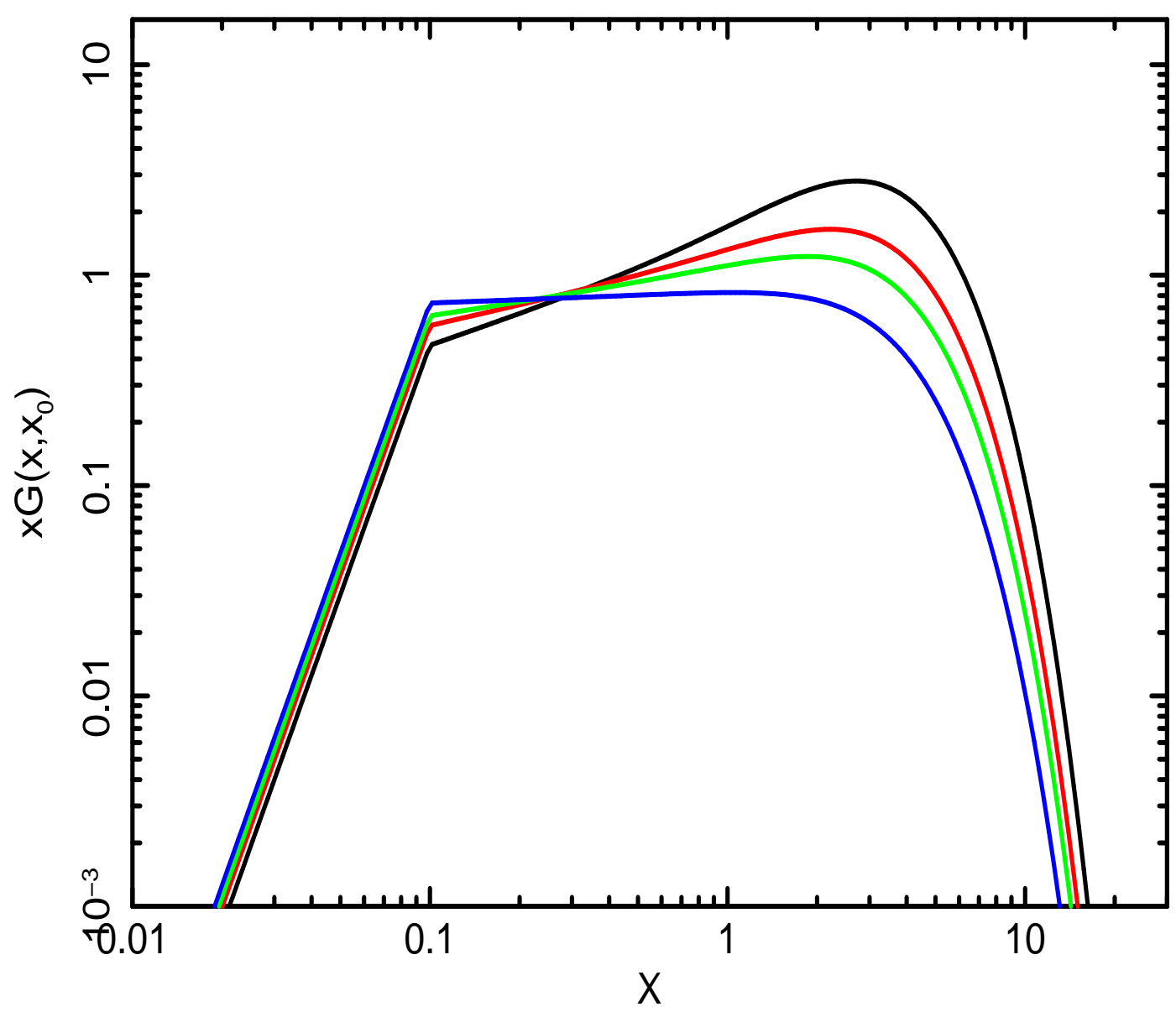

Fig. 2. - Evolution of the Green function (12) for the specific case of saturated Comptonization $(\gamma \sim 0)$ and different values of the bulk parameter $\delta$. Black, red, green and blue correspond to $\delta=0.5,0.7,0.8$ and 0.95 respectively. The horizontal scale is defined in units $x \equiv E / k T_{\mathrm{e}}$. 


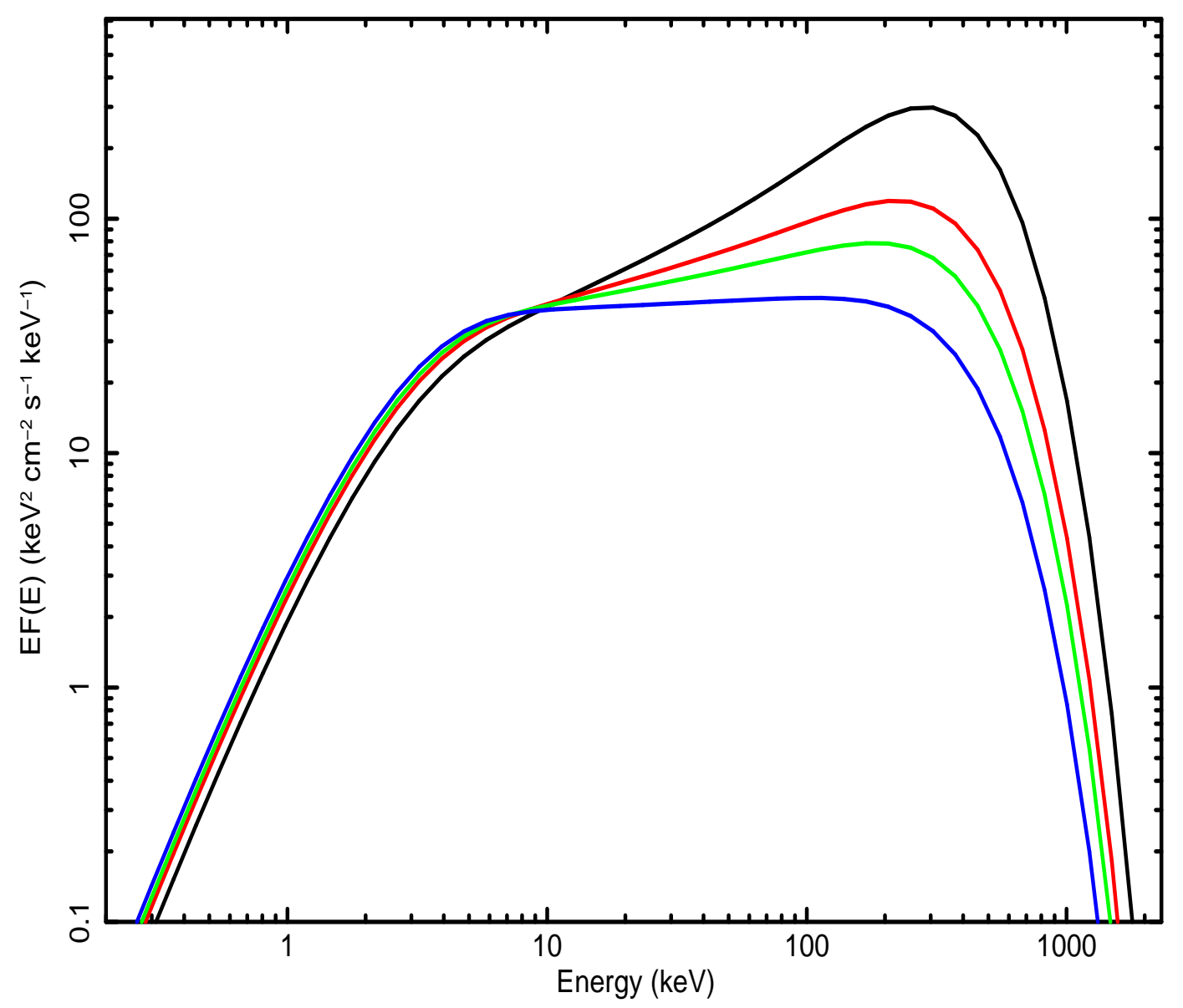

Fig. 3.- Saturated Comptonization spectra $(\gamma \sim 0)$ modified by the presence of subrelativitic bulk motion obtained from the numerical convolution of the Green function (12) with a blackbody spectrum of temperature $k T_{\mathrm{bb}}=1 \mathrm{keV}$. Different colors represent different values of the bulk parameter $\delta$ according to Fig. 2, A plasma temperature of $k T_{\mathrm{e}}=100 \mathrm{keV}$ is assumed. 


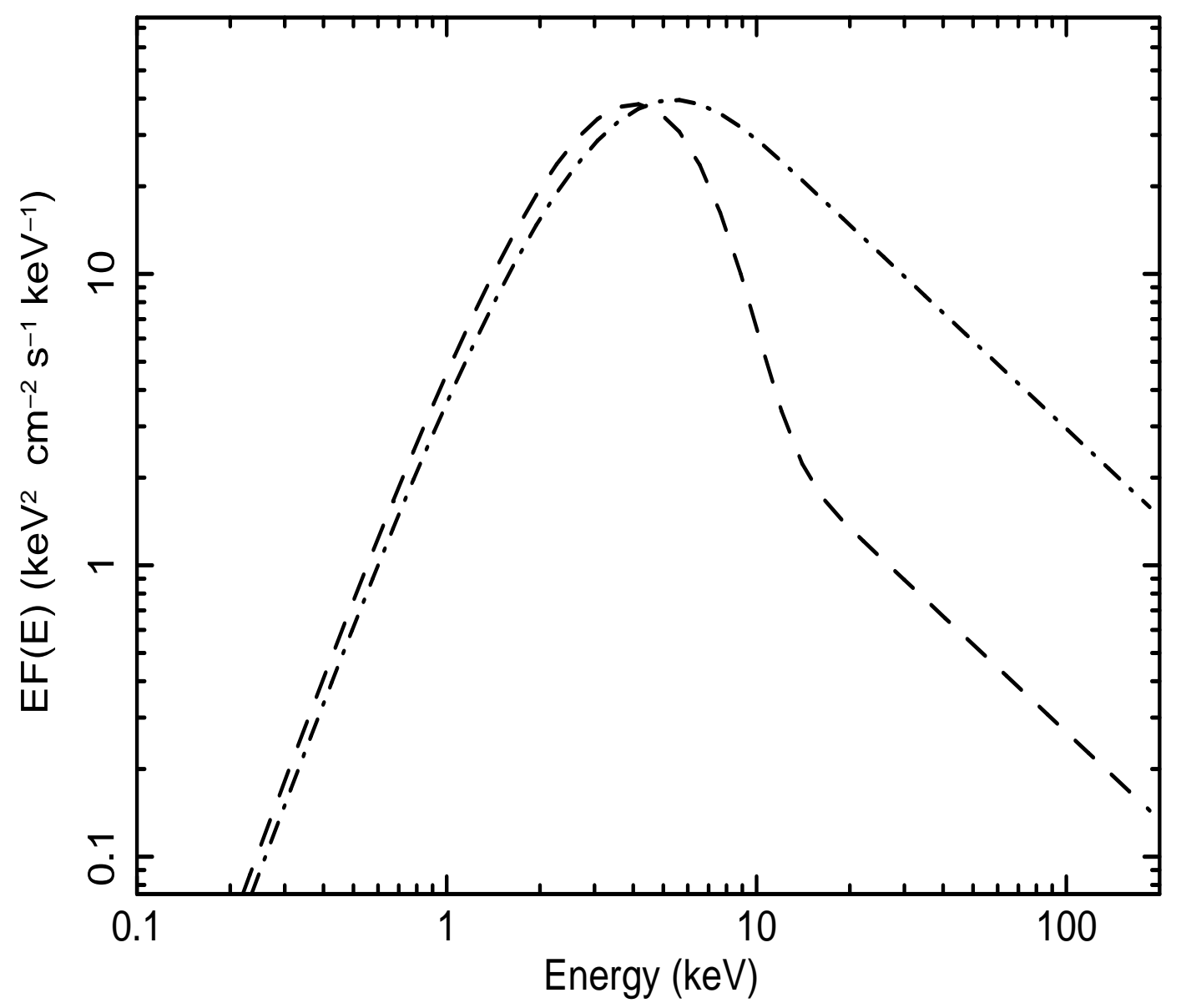

Fig. 4.- Emergent spectra obtained as the sum of a BB spectrum plus its convolution with a broken power-law Green function (see Eq. 18) and with weighting factors A $\gg 1$ (dotted-dashed line) and $\mathrm{A}=0.1$ (dashed line), according to equation (17). In both cases the $\mathrm{BB}$ temperature is $1 \mathrm{keV}$ and the Green function spectral index $\alpha_{\mathrm{b}}=2$. 


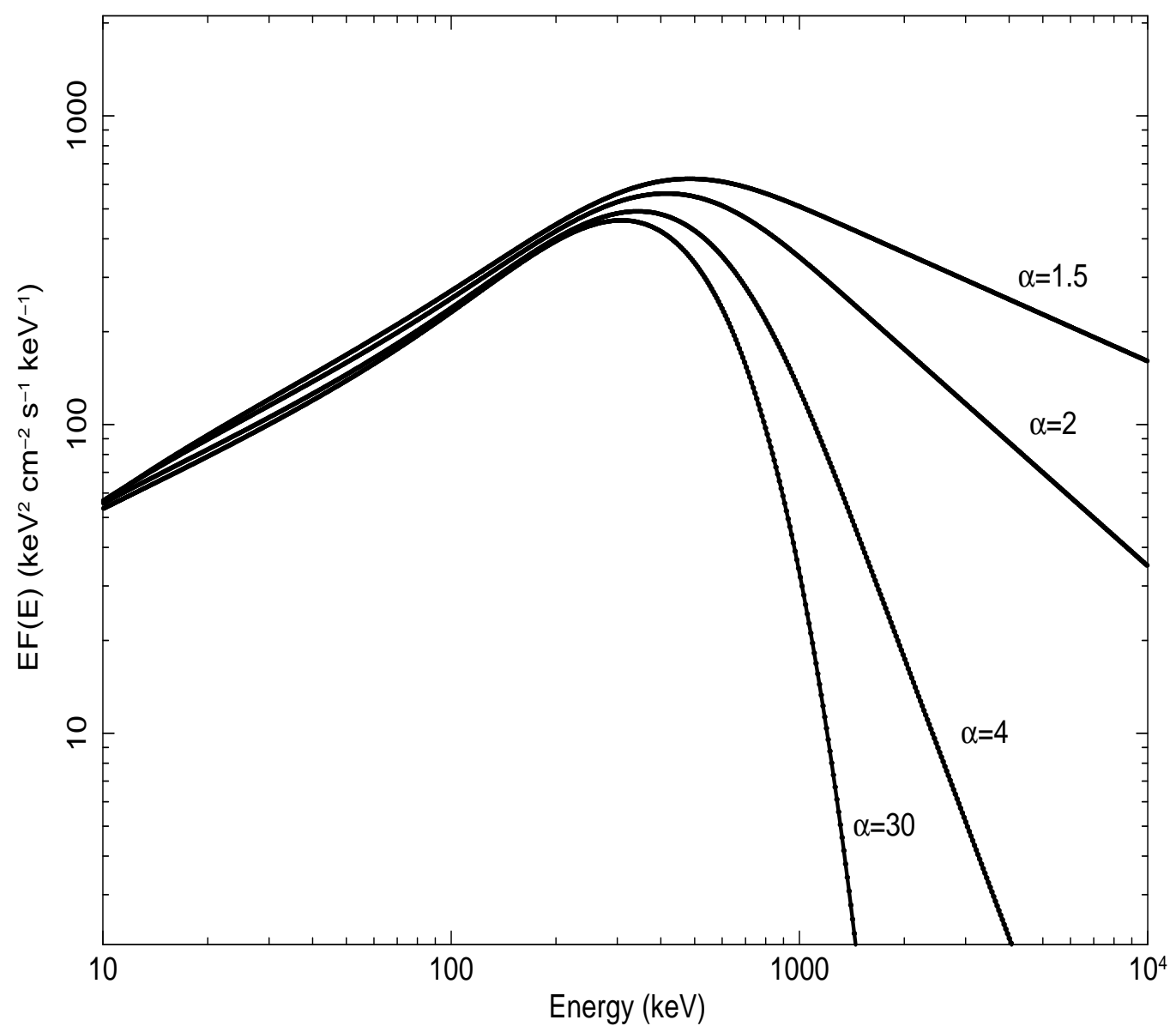

Fig. 5.- Emergent spectra obtained by the solution of Equations (8) and (19) for different values of the up-scattering Green function index $\alpha_{\mathrm{b}}$ determined by Eq. (18). Other parameters of the model are $k T_{\mathrm{bb}}=1 \mathrm{keV}, k T_{\mathrm{e}}=100 \mathrm{keV}, \delta=0.5, \beta_{0}=0.2$, and $\gamma \sim 0$. 

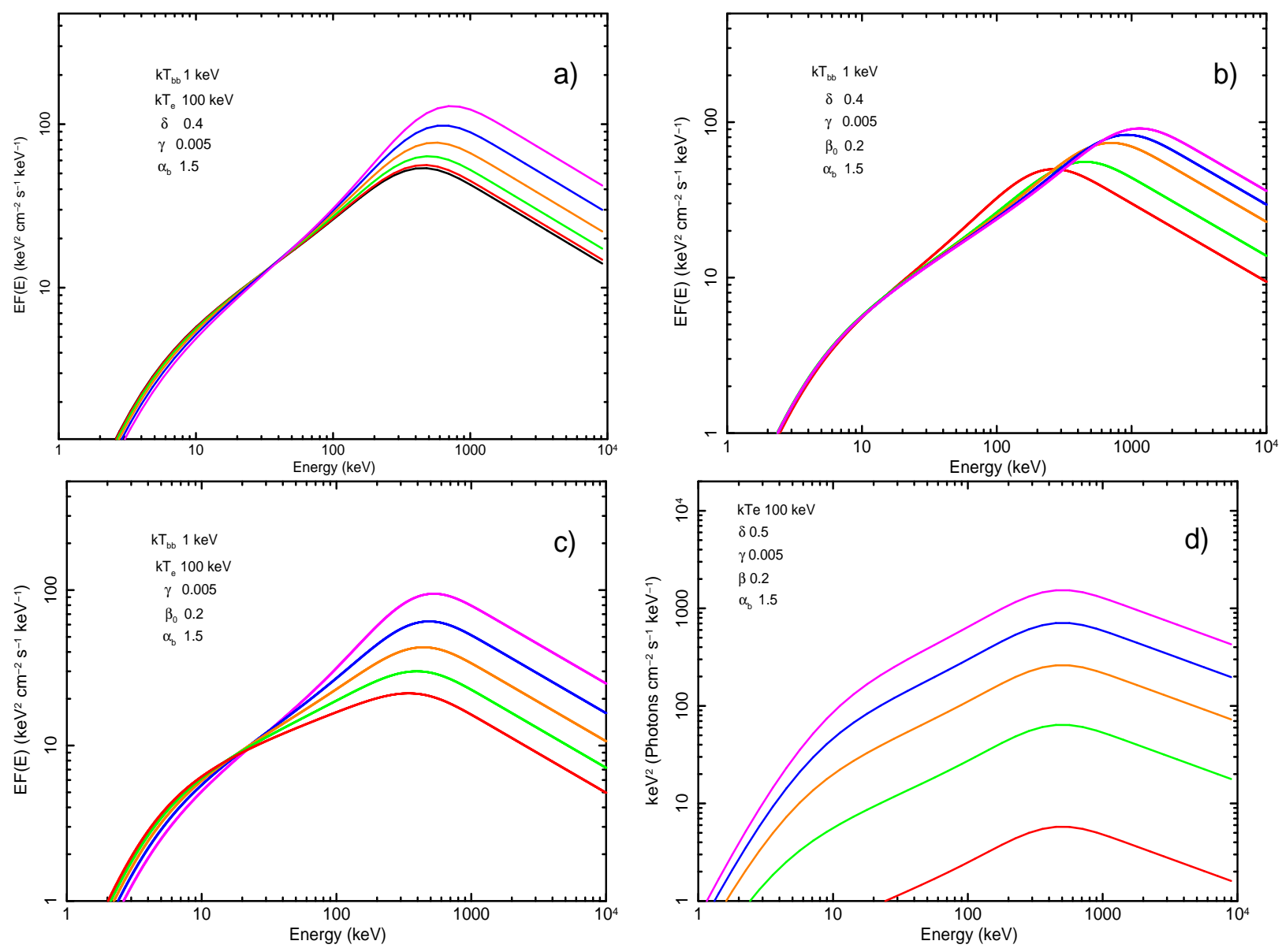

Fig. 6.- Simulated EF(E) spectra obtained as solutions of Eqs. (8) and (19) for different sets of the Compton parameters values. In the top left of each panel, the fixed parameters are reported. The low-energy steepening of the spectra is the transition towards the RayleighJeans region of the seed BB spectrum. Panel a): $\beta_{0}=0$ (black), 0.1 (red), 0.2 (green), 0.3 (orange), 0.4 (blue), 0.5 (pink). Panel b): $k T_{\mathrm{e}}=50 \mathrm{keV}$ (red), $100 \mathrm{keV}$ (green), $150 \mathrm{keV}$ (orange), $200 \mathrm{keV}$ (blue), $250 \mathrm{keV}$ (pink). Panel c): $\delta=0.8$ (red), 0.7 (green), 0.6 (orange), 0.5 (blue), 0.4 (pink). Panel d): $k T_{\mathrm{bb}}=0.5 \mathrm{keV}$ (red), $1 \mathrm{keV}$ (green), $1.5 \mathrm{keV}$ (orange), $2 \mathrm{keV}$ (blue), $2.55 \mathrm{keV}$ (pink). 


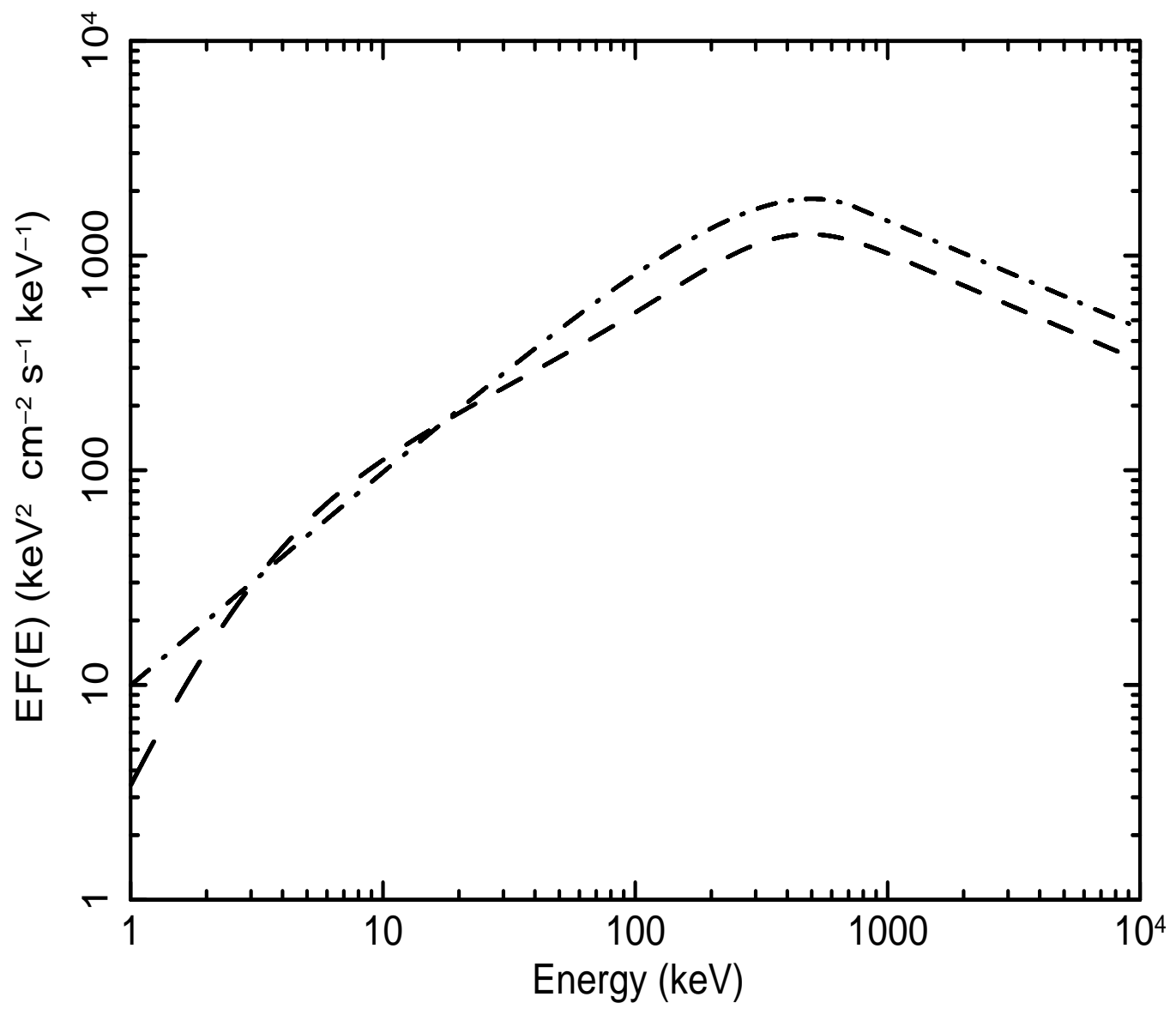

Fig. 7.- Comparison of the $\mathrm{EF}(\mathrm{E})$ spectra in the $1 \mathrm{keV}-10 \mathrm{MeV}$ energy range of our Comptonization model (dashed line) obtained from the solution of equations (8) and (19) and the Band function (dotted-dashed line). The parameters of the simulation are: $\Gamma_{1}=-1.5$, $\Gamma_{2}=-2.5$ and $E_{0}=350 \mathrm{keV}$ for the Band function, $k T_{\mathrm{bb}}=1 \mathrm{keV}, k T_{\mathrm{e}}=100 \mathrm{keV}, \delta=0.5$, $\gamma=5 \times 10^{-3}, \beta_{0}=0.4$ and $\alpha_{b}=1.5$, for the Comptonization spectrum. 

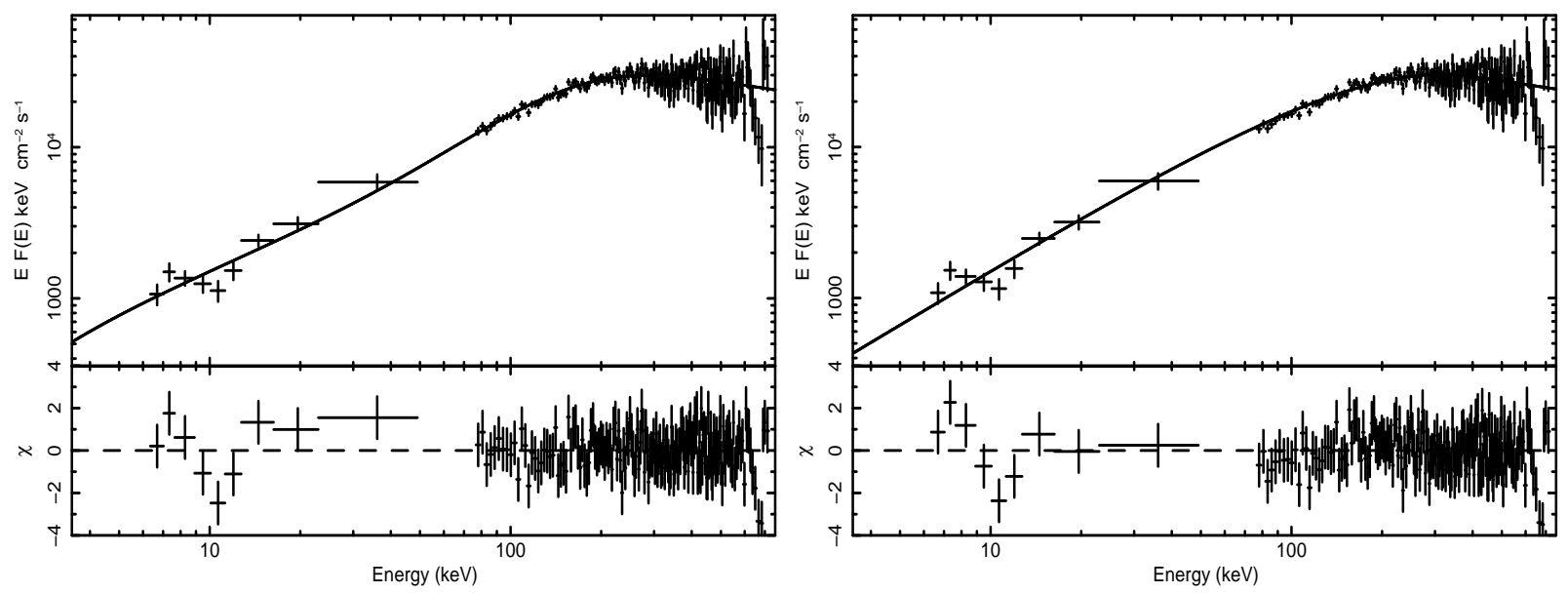

Fig. 8.- Unabsorbed EF(E) BeppoSAX/WFC+GRBM spectra of the prompt emission of GRB990705 ( $\mathrm{z}=0.842$; Amati 2006) and residuals between the data and the model in units of $\sigma$ using our photospheric model (left panel) and the Band function (right panel). Best-fit parameters for both cases are reported in Section 3 . 